\title{
Multiplicative renormalization of Yang-Mills theories in the background-field formalism
}

\author{
Igor A. Batalin ${ }^{1,2, a}$, Peter M. Lavrov ${ }^{2,3, b}$, Igor V. Tyutin ${ }^{1,2, c}$ \\ ${ }^{1}$ P.N. Lebedev Physical Institute, Leninsky Prospect 53, 119991 Moscow, Russia \\ 2 Tomsk State Pedagogical University, Kievskaya St. 60, 634061 Tomsk, Russia \\ ${ }^{3}$ National Research Tomsk State University, Lenin Av. 36, 634050 Tomsk, Russia
}

Received: 13 June 2018 / Accepted: 27 June 2018 / Published online: 12 July 2018

(C) The Author(s) 2018

\begin{abstract}
In the paper, within the background field method, the structure of renormalizations is studied as for Yang-Mills fields interacting with a multiplet of spinor fields. By extending the Faddeev-Popov action with extra fields and parameters, one is allowed to establish the multiplicative character of the renormalizability. The renormalization of the physical parameters is shown to be gauge-independent.
\end{abstract}

\section{Introduction}

When quantizing non-Abelian gauge field theories [1], whose gauge transformations form a group, one is naturally based on the Faddeev-Popov method [2]. It is a characteristic property of the Faddeev-Popov gauge-fixed action that the latter is invariant under global BRST supersymmetry [3,4], which, in turn, can be expressed in the form of the Zinn-Justin equation [5] for the Faddeev-Popov action. At the quantum level, the BRST symmetry as expressed in terms of the effective action, implies the Slavnov-Taylor identities [6,7] to hold. Further generalization as to the quantization of gauge theories, including the cases of field-dependent structure coefficients, as well as open and/or reducible gauge algebra, is described by the field-antifield BV formalism [8,9]. In that formalism, the effective action is BRST invariant by construction, and thus satisfies the master equation which provides for the gauge invariance of the physical sector of the theory $[8,9]$.

An interest to the gauge dependence problem did appear from the study of the effective potential, which appeared to be gauge-dependent in Yang-Mills theories with spontaneous breaking of the symmetry, when calculating physically-

\footnotetext{
a e-mail: batalin@1pi.ru

be-mail: lavrov@tspu.edu.ru

c e-mail: tyutin@1pi.ru
}

sensible results (the energy of the ground state, the masses of the physical particles, and so on) [10,11]. In Refs. [12,13] it was established that the energy of the ground state was gaugeindependent. Later, it was proved [14,15] that in Yang-Mills theories the dependence of gauge parameters in the effective action could be described in terms of gauge-invariant functional whose arguments (fields) were gauge-dependent (see also recent Refs. $[16,17]$ devoted to that problem as resolved via the procedure of redefinition of the field variables, found in $[14,15])$. Notice that in the general case of gauge theories, a variation in gauge condition is described in the form of certain change of the field variables (in terms of anticanonical transformations) $[18,19]$.

Although there are many papers devoted to various aspects of renormalizability of Yang-Mills theories, gauge dependence of renormalization constants has been studied explicitly only as for the gauge field sector [20]. In the present paper, within the background field formalism, it is studied a multiplicative renormalization procedure and gauge dependence as for Yang-Mills fields interacting with a multiplet of spinor fields. It is shown that renormalizations of physical parameters of the theory are gauge-independent.

The paper is organized as follows. In Sect. 2, it is discussed the action of Yang-Mills fields and spinor fields in the standard approach and in the background field method; it is also introduced extended action, which leads in the background field method to a multiplicative renormalizable theory of the fields considered; it is also studied the symmetry of the extended action. In Sect. 3, it is established the structure and the arbitrariness is described as for any local functional with the quantum numbers of the extended action that satisfies the same set of equations as the extended action. In Sect. 4, the equations are derived for the generating functional of vertexes (effective action), as a consequence at the quantum level, of the symmetry property of the extended action; and it is shown that the generating functional of vertexes satis- 
fies the same equations as the extended classical action. In Sect. 5, it is studied the renormalization procedure of the theory considered when using the loop expansion technique and the minimal subtraction scheme; and thus the multiplicative renormalizability of the theory is proved. In Sect. 6, the relations are found between the parameters of the renormalized action and the standard renormalization constants of fields and vertexes of the interaction, and renormalized physical parameters are shown to be gauge-independent. Concluding remarks are given in Sect. 7.

Condensed DeWitt's notations [21] are used through the paper. Functional derivatives with respect to field variables are understood as the left. Right derivatives of a quantity $f$ with respect to the variable $\varphi$ are denoted as $f \frac{\overleftarrow{\delta}}{\delta \varphi}$.

\section{Extended action for Yang-Mills theories}

Let us consider a gauge theory of non-abelian vector fields $A_{\mu}^{\alpha}=A_{\mu}^{\alpha}(x)$ and spinor fields $\psi_{j}=\psi_{j}(x), \bar{\psi}_{j}=\bar{\psi}_{j}(x)$ in the $D=4$ Minkowski space-time with the action

$$
\begin{aligned}
\mathcal{S}_{Y M}(A, \Psi)= & \int d x\left(-\frac{1}{4} G_{\mu \nu}^{\alpha}(A) G_{\mu \nu}^{\alpha}(A)\right. \\
& \left.+i \bar{\psi}_{j} \gamma^{\mu} D_{\psi \mu j k}(A) \psi_{k}-m \bar{\psi}_{j} \psi_{j}\right),
\end{aligned}
$$

where the notations

$$
\begin{gathered}
G_{\mu \nu}^{\alpha}(A)=\partial_{\mu} A_{v}^{\alpha}-\partial_{\nu} A_{\mu}^{\alpha}+g f^{\alpha \beta \gamma} A_{\mu}^{\beta} A_{\nu}^{\gamma}, \\
D_{\psi \mu j k}(A)=\partial_{\mu} \delta_{j k}+g t_{j k}^{\alpha} A_{\mu}^{\alpha}, \quad \Psi=\{\psi, \bar{\psi}\}
\end{gathered}
$$

are used. In Eqs. (2.1), (2.2) $f^{\alpha \beta \gamma}$ are structure coefficients of a simple compact gauge Lie group, $t^{\alpha}=\left\{t_{j k}^{\alpha}\right\}$ are generators of gauge transformations in sector of spinor fields satisfying the properties,

$\left[t^{\alpha}, t^{\beta}\right]=f^{\alpha \beta \gamma} t^{\gamma}, \quad\left(t^{\gamma}\right)^{+}=-t^{\gamma}, \quad\left[\gamma^{\mu}, t^{\alpha}\right]=0$.

Here $\gamma^{\mu}$ are the Dirac matrices, $g$ and $m$ are the coupling constant of gauge interaction and the mass parameter of spinor field, respectively. The action (2.1) is invariant under gauge transformations with gauge parameters $\omega_{\alpha}=\omega_{\alpha}(x)$, $\delta_{\omega} \mathcal{S}_{Y M}(A, \Psi)=0$,

$$
\begin{aligned}
& \delta_{\omega} A_{\mu}^{\alpha}=\left(\partial_{\mu} \delta_{\alpha \beta}+g f^{\alpha \sigma \beta} A_{\mu}^{\sigma}\right) \omega_{\beta}=D_{\mu}^{\alpha \beta}(A) \omega_{\beta}, \\
& \delta_{\omega} \psi_{j}=-g t_{j k}^{\beta} \psi_{k} \omega_{\beta}, \quad \delta_{\omega} \bar{\psi}_{j}=g \bar{\psi}_{k} t_{k j}^{\beta} \omega_{\beta} .
\end{aligned}
$$

The corresponding Faddeev-Popov action [2] $S_{F P}^{(1)}=$ $S_{F P}^{(1)}(A, \Psi, C, \bar{C}, B, \xi)$ in the Feynman gauge has the form

$$
\begin{aligned}
S_{F P}^{(1)}= & \mathcal{S}_{Y M}(A, \Psi)+\int d x\left(\bar{C}^{\alpha} \partial_{\mu} D_{\mu}^{\alpha \beta}(A) C^{\beta}\right. \\
& \left.+B^{\alpha} \partial_{\mu} A_{\mu}^{\alpha}+(\xi / 2) B^{\alpha} B^{\alpha}\right),
\end{aligned}
$$

where $\xi$ is a constant gauge parameter, $C^{\alpha}=C^{\alpha}(x), \bar{C}^{\alpha}=$ $\bar{C}^{\alpha}(x)$ represent the Faddeev-Popov ghost fields, $B^{\alpha}=$ $B^{\alpha}(x)$ are auxiliary fields introducing a gauge fixing condition. The action (2.5) is invariant under global supersymmetry (BRST symmetry) $[3,4]$,

$$
\begin{aligned}
& \delta_{\lambda} A_{\mu}^{\alpha}=D_{\mu}^{\alpha \beta}(A) C^{\beta} \lambda, \quad \delta_{\lambda} \psi_{j}=-g t_{j k}^{\alpha} \psi_{k} C^{\alpha} \lambda, \\
& \delta_{\lambda} \bar{\psi}_{j}=g \bar{\psi}_{k} t_{k j}^{\alpha} C^{\alpha} \lambda, \\
& \delta_{\lambda} C^{\alpha}=\frac{g}{2} f^{\alpha \beta \gamma} C^{\beta} C^{\gamma} \lambda, \quad \delta_{\lambda} \bar{C}^{\alpha}=B^{\alpha} \lambda, \quad \delta_{\lambda} B^{\alpha}=0,
\end{aligned}
$$

where $\lambda$ is a constant anticommuting parameter.

In the background field formalism [22,23] a gauge field $A_{\mu}^{\alpha}$ entering the classical action (2.1) is replaced by $A_{\mu}^{\alpha}+\mathcal{B}_{\mu}^{\alpha}$,

$\mathcal{S}_{Y M}(A, \Psi) \rightarrow \mathcal{S}_{Y M}(A+\mathcal{B}, \Psi)$,

where $\mathcal{B}_{\mu}^{\alpha}$ is considered as an external vector field. The Faddeev-Popov action is constructed by using the modified Feynman gauge (the background gauge condition), and reads

$$
\begin{aligned}
S_{F P}^{(2)}= & \mathcal{S}_{Y M}(A+\mathcal{B}, \Psi)+\int d x\left(\bar{C}^{\alpha} D_{\mu}^{\alpha \gamma}(\mathcal{B}) D_{\mu}^{\gamma \beta}(A+\mathcal{B}) C^{\beta}\right. \\
& \left.+B^{\alpha} D_{\mu}^{\alpha \beta}(\mathcal{B}) A_{\mu}^{\alpha}+(\xi / 2) B^{\alpha} B^{\alpha}\right) .
\end{aligned}
$$

This action is invariant under BRST transformations of the form (2.6) with the following modification of the transformation law in the gauge field sector,

$\delta_{\lambda} A_{\mu}^{\alpha}=D_{\mu}^{\alpha \beta}(A+\mathcal{B}) C^{\beta} \lambda$.

The invariance property of Faddeev-Popov actions (2.5) and (2.8) under BRST transformations can be described in the form of non-linear functional equations for the extended action $\mathcal{S}_{\text {ext }}$ with the help of additional variables (antifields) $A_{\mu}^{* \alpha}, \psi_{j}^{*}, \bar{\psi}_{j}^{*}, C^{* \alpha}, \bar{C}^{* \alpha}$, being sources to the generators of BRST transformations,

$\mathcal{S}_{\text {ext }}^{(a)}=S_{F P}^{(a)}+\int d x\left(Q^{*} \mathcal{R}_{Q}^{(a)}+\bar{C}^{* \alpha} B^{\alpha}\right), \quad a=1,2$,

where $Q$ means the set of the fields $\left\{A_{\mu}^{\alpha}, \psi_{j}, \bar{\psi}_{j}, C^{\alpha}\right\}$ and the symbol $Q^{*}$ is used to indicate the set of the corresponding antifields for fields $Q$, wherein the BRST transformations (2.4), (2.6) are presented as $\delta_{\lambda} Q=\mathcal{R}_{Q}^{(a)} \lambda, a=1,2$. Then, as a consequence of the BRST symmetry, the actions $\mathcal{S}_{\text {ext }}^{(a)}$ satisfy the master-equation

$\int d x\left(\mathcal{S}_{\text {ext }}^{(a)} \frac{\overleftarrow{\delta}}{\delta Q} \frac{\delta}{\delta Q^{*}} \mathcal{S}_{\text {ext }}^{(a)}+\mathcal{S}_{\text {ext }}^{(a)} \frac{\overleftarrow{\delta}}{\delta \bar{C}} \frac{\delta}{\delta \bar{C}^{*}} \mathcal{S}_{\text {ext }}^{(a)}\right)=0, \quad a=1,2$ 
To study the structure of renormalizations it is convenient to extend the original set of the variables with extra fields and auxiliary quantities. An initial action, we proceed from, when studying the structure of renormalizations and dependence of renormalization constants on gauge fixing is the extended action $S_{\text {ext }}=S_{\text {ext }}\left(Q, Q^{*}, \bar{C}, B, \mathcal{B}, \xi, \theta, \chi\right)$,

$$
\begin{aligned}
S_{\text {ext }}= & S_{Y M}(A+\mathcal{B}, \Psi)+\int d x Q^{*} \mathcal{R}_{Q} \\
& +\int d x\left(\bar{C}^{\alpha} D_{\mu}^{\alpha \gamma}(\mathcal{B}) D_{\mu}^{\gamma \beta}(A+\mathcal{B}) C^{\beta}\right. \\
& \left.+B^{\alpha} D_{\mu}^{\alpha \beta}(\mathcal{B}) A_{\mu}^{\beta}+(\xi / 2) B^{\alpha} B^{\alpha}\right) \\
& +\int d x\left(\theta_{\mu}^{\alpha}\left[D_{\mu}^{\alpha \beta}(A+\mathcal{B}) \bar{C}^{\beta}-A_{\mu}^{* \alpha}\right]\right. \\
& +\chi\left[\left(A_{\mu}^{* \alpha}-D_{\mu}^{\alpha \beta}(\mathcal{B}) \bar{C}^{\beta}\right) A_{\mu}^{\alpha}+C^{* \alpha} C^{\alpha}+\psi_{j}^{*} \psi_{j}\right. \\
& \left.\left.+\bar{\psi}_{j}^{*} \bar{\psi}_{j}\right]\right)
\end{aligned}
$$

where $\theta_{\mu}^{\alpha}=\theta_{\mu}^{\alpha}(x)$ are anticommuting extra fields and $\chi$ is a constant nilpotent parameter. ${ }^{1}$

The action (2.12) is invariant $\left(\delta S_{\text {ext }}=0\right)$ under the following transformations of the quantities entered,

$$
\begin{aligned}
\delta A_{\mu}^{\alpha}= & D_{\mu}^{\alpha \beta}(A+\mathcal{B}) C^{\beta}+\theta_{\mu}^{\alpha}=\frac{\delta}{\delta A_{\mu}^{* \alpha}} S_{\mathrm{ext}}+\chi A_{\mu}^{\alpha} \\
\delta B^{\alpha}= & -\frac{1}{\xi}\left[D_{\mu}^{\alpha \gamma}(\mathcal{B}) D^{\gamma \beta}(A+\mathcal{B}) C^{\beta}+D_{\mu}^{\alpha \beta}(A+\mathcal{B}) \theta_{\mu}^{\beta}\right. \\
& \left.+\chi D_{\mu}^{\alpha \beta}(\mathcal{B}) A_{\mu}^{\beta}\right]-\chi B^{\alpha} \\
= & -\frac{1}{\xi} \frac{\delta}{\delta \bar{C}^{\alpha}} S_{\mathrm{ext}}-\chi B^{\alpha} \\
\delta C^{\alpha}= & \frac{g}{2} f^{\alpha \beta \gamma} C^{\beta} C^{\gamma}=\frac{\delta}{\delta C^{* \alpha}} S_{\mathrm{ext}}-\chi C^{\alpha} \\
\delta \bar{C}^{\alpha}= & -\frac{1}{\xi} D_{\mu}^{\alpha \beta}(\mathcal{B}) A_{\mu}^{\beta}+\chi \bar{C}^{\alpha}=-\frac{1}{\xi} \frac{\delta}{\delta B^{\alpha}} S_{\mathrm{ext}}+B^{\alpha} \\
& +\chi \bar{C}^{\alpha}, \\
\delta \psi_{j}= & -g t_{j k}^{\alpha} \psi_{k} C^{\alpha}=\frac{\delta}{\delta \psi_{j}^{*}} S_{\mathrm{ext}}-\chi \psi_{j} \\
\delta \bar{\psi}_{j}= & g \bar{\psi}_{k} t_{k j}^{\alpha} C^{\alpha}=\frac{\delta}{\delta \bar{\psi}_{j}^{*}} S_{\mathrm{ext}}-\chi \bar{\psi}_{j} \\
\delta A_{\mu}^{* \alpha}= & \chi A_{\mu}^{* \alpha}, \quad \delta C^{* \alpha}=-\chi C^{* \alpha}, \quad \delta \psi_{j}^{*}=-\chi \psi_{j}^{*} \\
& \delta \bar{\psi}_{j}^{*}=-\chi \bar{\psi}_{j}^{*}, \\
\delta \xi= & 2 \xi \chi, \quad \delta \mathcal{B}_{\mu}^{\alpha}=-\theta_{\mu}^{\alpha}, \quad \delta \theta_{\mu}^{\alpha}=0
\end{aligned}
$$

\footnotetext{
1 These extra variables have been used first in Ref. [20].
}

Due to the variations (2.13)-(2.20), the invariance condition of the action rewrites

$$
\begin{aligned}
& \int d x\left(S_{\mathrm{ext}} \frac{\overleftarrow{\delta}}{\delta Q} \frac{\delta}{\delta Q^{*}} S_{\mathrm{ext}}-B \frac{\delta}{\delta \bar{C}} S_{\mathrm{ext}}-\theta \frac{\delta}{\delta \mathcal{B}} S_{\mathrm{ext}}\right) \\
& +\chi \int d x\left[Q \frac{\delta}{\delta Q}-Q^{*} \frac{\delta}{\delta Q^{*}}-\bar{C} \frac{\delta}{\delta \bar{C}}-B \frac{\delta}{\delta B}\right] S_{\mathrm{ext}} \\
& +2 \chi \xi \frac{\partial}{\partial \xi} S_{\mathrm{ext}}=0
\end{aligned}
$$

Also, the action (2.12) satisfies the equation

$S_{\mathrm{ext}} \overleftarrow{H^{\alpha}} \omega_{\alpha}=0$

where the notation

$$
\begin{aligned}
& \overleftarrow{H^{\alpha}} \omega_{\alpha}=\int d x\left\{\left[\frac{\overleftarrow{\delta}}{\delta \mathcal{B}_{\mu}^{\beta}} D_{\mu}^{\beta \alpha}(\mathcal{B})\right.\right. \\
& +g f^{\beta \gamma \alpha}\left(\frac{\overleftarrow{\delta}}{\delta A_{\mu}^{\beta}} A_{\mu}^{\gamma}+\frac{\overleftarrow{\delta}}{\delta B^{\beta}} B^{\gamma}\right) \\
& +g f^{\beta \gamma \alpha}\left(\frac{\overleftarrow{\delta}}{\delta C^{\beta}} C^{\gamma}+\frac{\overleftarrow{\delta}}{\delta \bar{C}^{\beta}} \bar{C}^{\gamma}+\frac{\overleftarrow{\delta}}{\delta A_{\mu}^{* \beta}} A_{\mu}^{* \gamma}\right. \\
& \left.+\frac{\overleftarrow{\delta}}{\delta C^{* \beta}} C^{* \gamma}+\frac{\overleftarrow{\delta}}{\delta \theta_{\mu}^{\beta}} \theta_{\mu}^{\gamma}\right) \\
& -g t_{j k}^{\alpha}\left(\frac{\overleftarrow{\delta}}{\delta \psi_{j}} \psi_{k}+\frac{\overleftarrow{\delta}}{\delta \bar{\psi}_{j}^{*}} \bar{\psi}_{k}^{*}\right) \\
& \left.\left.+g\left(\frac{\overleftarrow{\delta}}{\delta \bar{\psi}_{j}} \bar{\psi}_{k}+\frac{\overleftarrow{\delta}}{\delta \psi_{j}^{*}} \psi_{k}^{*}\right) t_{k j}^{\alpha}\right] \omega_{\alpha}\right\}
\end{aligned}
$$

is used for the operator describing the gauge transformations of the variables $\mathcal{B}_{\mu}, \psi, \bar{\psi}$ and simultaneously the tensor transformation of fields and antifields $A_{\mu}, C, \bar{C}, B, \theta_{\mu}, A_{\mu}^{*}, \psi^{*}$, $\bar{\psi}^{*}, C^{*}$. Finally, we notice that the action (2.12) satisfies the two important relations linear in fields $A_{\mu}, B$ and also in derivatives of variables $B, \bar{C}, A_{\mu}^{*}$,

$$
\begin{aligned}
& \frac{\delta}{\delta B^{\alpha}} S_{\mathrm{ext}}=D_{\mu}^{\alpha \beta}(\mathcal{B}) A_{\mu}^{\beta}+\xi B^{\alpha} \\
& D_{\mu}^{\alpha \beta}(\mathcal{B}) \frac{\delta}{\delta A_{\mu}^{* \beta}} S_{\mathrm{ext}}-\frac{\delta}{\delta \bar{C}^{\alpha}} S_{\mathrm{ext}}=-g f^{\alpha \beta \gamma} A_{\mu}^{\beta} \theta_{\mu}^{\gamma}
\end{aligned}
$$

The Eq. (2.25) means that the action $S_{\text {ext }}$ (2.12) depends on variables $A_{\mu}^{* \alpha}$ " $Z$ ", " $p$ " $\bar{C}^{\alpha}$ in combination $A_{\mu}^{* \alpha}-D_{\mu}^{\alpha \beta}(\mathcal{B}) \bar{C}^{\beta}$ only when $\theta_{\mu}^{\beta}=0$.

We give the table of "quantum" numbers of fields, antifields, auxiliary fields and constant quantities which have been used in constructing $S_{\text {ext }}$ : 


\begin{tabular}{lllllllllllll}
\hline Quantity & $A, \mathcal{B}$ & $\psi, \bar{\psi}$ & $\mathrm{C}, \bar{C}$ & $\mathrm{~B}$ & $\xi$ & $\theta$ & $A^{*}$ & $\psi^{*}, \bar{\psi}^{*}$ & $C^{*}$ & $\mathrm{dx}$ & $\partial_{x}$ & $\chi$ \\
\hline$\varepsilon$ & 0 & 1 & 1 & 0 & 0 & 1 & 1 & 0 & & 0 & 0 & 0 \\
gh & 0 & 0 & $1,-1$ & 0 & 0 & 1 & -1 & -1 & -2 & 0 & 0 & 1 \\
$\operatorname{dim}$ & 1 & $3 / 2$ & 1 & 2 & 0 & 2 & 2 & $3 / 2$ & 2 & -4 & 1 \\
$\varepsilon_{f}$ & 0 & $1,-1$ & 0 & 0 & 0 & 0 & 0 & $-1,1$ & 0 & 0 & 0 & 0 \\
\hline
\end{tabular}

where " $\varepsilon$ " describes the Grassmann parity, the symbol "gh" is used for the ghost number, "dim" denotes the canonical dimension and " $\varepsilon$ " means the fermion number. Using this table of "quantum" numbers it is easy to establish quantum numbers of any quantities found in the text.

\section{General structure of renormalized action}

It is to be proved below that the renormalizable action is a local functional of field variables, carries the quantum number of the action $\mathcal{S}_{\text {ext }}$ (2.12), and satisfies the same Eqs. (2.21)-(2.25) as the action $S_{\text {ext }}$. In that Section we will find the general solution to the Eqs. (2.21)-(2.25) under the extra conditions mentioned.

So, let

$P=\int d x P(x)$

where $P(x)$ is a local polynomial in all variables $Q, Q^{*}, \bar{C}$, $B, \mathcal{B}, \xi, \theta, \chi$ with $\operatorname{dim}(P(x))=4$. Require the functional $P$ to satisfy the Eqs. (2.21)-(2.25) with substitution $S_{\text {ext }} \rightarrow P$, and let $P$ be of the form

$P=P_{00}+P^{(1)}+\chi P^{(2)}$,

where

$$
\begin{aligned}
P_{00}= & \int d x\left(B^{\alpha} D_{\mu}^{\alpha \beta}(\mathcal{B}) A_{\mu}^{\beta}+\frac{\xi}{2} B^{\alpha} B^{\alpha}\right. \\
& \left.+g \theta_{\mu}^{\alpha} f^{\alpha \beta \gamma} A_{\mu}^{\beta} \bar{C}^{\gamma}\right), \\
\varepsilon\left(P^{(1)}\right)= & 0, \quad \operatorname{gh}\left(P^{(1)}\right)=0, \\
\operatorname{dim}\left(P^{(1)}\right)= & 0, \quad \varepsilon_{f}\left(\left(P^{(1)}\right)\right)=0, \\
\varepsilon\left(P^{(2)}\right)= & 1, \quad \operatorname{gh}\left(P^{(2)}\right)=-1, \\
\operatorname{dim}\left(P^{(2)}\right)= & -1, \quad \varepsilon_{f}\left(\left(P^{(2)}\right)\right)=0,
\end{aligned}
$$

and the functionals $P^{(1)}$ and $P^{(2)}$ do not depend on $\chi$. It follows from the Eq. (2.24) for $P$, and representation (3.3) that $P^{(1)}$ and $P^{(2)}$ do not depend on $B^{\alpha}$, " $Z$ ", " $p$ ",

$P^{(k)}=P^{(k)}\left(Q, Q^{*}, \bar{C}, \mathcal{B}, \xi, \theta\right), \quad k=1,2$,

By introducing new variables $\mathcal{A}_{\mu}^{* \alpha}(x)$,

$\mathcal{A}_{\mu}^{* \alpha}=A_{\mu}^{* \alpha}-D_{\mu}^{\alpha \beta}(\mathcal{B}) \bar{C}^{\beta}, \quad \mathcal{A}_{\mu}^{\alpha}=A_{\mu}^{\alpha}$, we define new functionals $\tilde{P}^{(k)}$ by the rule

$$
\begin{aligned}
\tilde{P}^{(k)} & =\tilde{P}^{(k)}\left(\Omega, \Omega^{*}, \mathcal{B}, \bar{C}, \xi, \theta\right) \\
& =\left.P^{(k)}\left(Q, Q^{*}, \mathcal{B}, \bar{C}, \xi, \theta\right)\right|_{A^{*} \rightarrow \mathcal{A}^{*}+D(\mathcal{B}) \bar{C}}, \quad k=1,2,
\end{aligned}
$$

to find that $\tilde{P}^{(k)}$ do not depend on the fields $\bar{C}^{\alpha}$,

$\tilde{P}^{(k)}=\tilde{P}^{(k)}\left(\Omega, \Omega^{*}, \mathcal{B}, \xi, \theta\right)$

In the relations (3.8) and (3.9) the following notations

$\Omega=\{\mathcal{A}, \psi, \bar{\psi}, C\}, \mathcal{A}=A, \Omega^{*}=\left\{\mathcal{A}^{*}, \psi^{*}, \bar{\psi}^{*}, C^{*}\right\}$

are used.

Independence of functionals $\tilde{P}^{(k)}$ of the fields $\bar{C}^{\alpha}$ and relations

$$
\begin{aligned}
& P^{(k)} \int d x\left[\frac{\overleftarrow{\delta}}{\delta \mathcal{B}_{\mu}^{\beta}} D_{\mu}^{\beta \alpha}(\mathcal{B})+g f^{\beta \gamma \alpha}\left(\frac{\overleftarrow{\delta}}{\delta \bar{C}^{\beta}} \bar{C}^{\gamma}+\frac{\overleftarrow{\delta}}{\delta A_{\mu}^{* \beta}} A_{\mu}^{* \gamma}\right)\right] \\
& =\tilde{P}^{(k)} \int d x\left[\frac{\overleftarrow{\delta}}{\delta \mathcal{B}_{\mu}^{\beta}} D_{\mu}^{\beta \alpha}(\mathcal{B})+g f^{\beta \gamma \alpha} \frac{\overleftarrow{\delta}}{\delta \mathcal{A}_{\mu}^{* \beta}} \mathcal{A}_{\mu}^{* \gamma}\right]
\end{aligned}
$$

allow one to write down the following set of equations as for $\tilde{P}^{(k)}$,

$$
\begin{aligned}
\int d x\left[\tilde{P}^{(1)} \frac{\overleftarrow{\delta}}{\delta \Omega} \frac{\delta}{\delta \Omega^{*}} \tilde{P}^{(1)}-\theta_{\mu}^{\alpha} \frac{\delta}{\delta \mathcal{B}_{\mu}^{\alpha}} \tilde{P}^{(1)}\right]=0 \\
2 \xi \frac{\partial}{\partial \xi} \tilde{P}^{(1)}=\int d x\left[\tilde{P}^{(1)}\left(\frac{\overleftarrow{\delta}}{\delta \Omega} \frac{\delta}{\delta \Omega^{*}}-\frac{\overleftarrow{\delta}}{\delta \Omega^{*}} \frac{\delta}{\delta \Omega}\right) \tilde{P}^{(2)}\right. \\
\left.-\theta_{\mu}^{\alpha} \frac{\delta}{\delta \mathcal{B}_{\mu}^{\alpha}} \tilde{P}^{(2)}\right] \\
+\int d x\left[\left(\Omega^{*} \frac{\delta}{\delta \Omega^{*}}-\Omega \frac{\delta}{\delta \Omega}\right) \tilde{P}^{(1)}\right]
\end{aligned}
$$

$\tilde{P}^{(k)} \overleftarrow{\tilde{h}^{\alpha}} \omega_{\alpha}=0, k=1,2$ 
where

$$
\begin{aligned}
\overleftarrow{\overleftarrow{h}^{\alpha}} \omega_{\alpha}= & \int d x\left\{\left[\frac{\overleftarrow{\delta}}{\delta \mathcal{B}_{\mu}^{\beta}} D_{\mu}^{\beta \alpha}(\mathcal{B})\right.\right. \\
& +g f^{\beta \gamma \alpha}\left(\frac{\overleftarrow{\delta}}{\delta \mathcal{A}_{\mu}^{\beta}} \mathcal{A}_{m \mid \mu}^{\gamma}+\frac{\overleftarrow{\delta}}{\delta C^{\beta}} C^{\gamma}\right) \\
& +g f^{\beta \gamma \alpha}\left(\frac{\overleftarrow{\delta}}{\delta \mathcal{A}_{\mu}^{* \beta}} \mathcal{A}_{\mu}^{* \gamma}+\frac{\overleftarrow{\delta}}{\delta C^{* \beta}} C^{* \gamma}+\frac{\overleftarrow{\delta}}{\delta \theta^{\beta}} \theta_{\mu}^{\gamma}\right) \\
& -g t_{j k}^{\alpha}\left(\frac{\overleftarrow{\delta}}{\delta \psi_{j}} \psi_{k}\right. \\
& \left.\left.\left.+\frac{\overleftarrow{\delta}}{\delta \bar{\psi}_{j}^{*}} \bar{\psi}_{k}^{*}\right)+g\left(\frac{\overleftarrow{\delta}}{\delta \bar{\psi}_{j}} \bar{\psi}_{k}+\frac{\overleftarrow{\delta}}{\delta \psi_{j}^{*}} \psi_{k}^{*}\right) t_{k j}^{\alpha}\right] \omega_{\alpha}\right\}
\end{aligned}
$$

When studying the structure of functionals and further investigating it appears useful a consequence of the Eq. (3.13) at $\omega_{\alpha}=$ const,

$\tilde{P}^{(k)} \overleftarrow{T^{\alpha}}=0, \quad k=1,2$

where

$$
\begin{aligned}
\overleftarrow{T^{\alpha}}= & \int d x\left\{f^{\beta \gamma \alpha}\left(\frac{\overleftarrow{\delta}}{\delta \mathcal{B}_{\mu}^{\beta}} \mathcal{B}_{\mu}^{\gamma}+\frac{\overleftarrow{\delta}}{\delta \mathcal{A}_{\mu}^{\beta}} \mathcal{A}_{\mu}^{\gamma}+\frac{\overleftarrow{\delta}}{\delta C^{\beta}} C^{\gamma}\right)\right. \\
& +f^{\beta \gamma \alpha}\left(\frac{\overleftarrow{\delta}}{\delta \mathcal{A}_{\mu}^{* \beta}} \mathcal{A}_{\mu}^{* \gamma}+\frac{\overleftarrow{\delta}}{\delta C^{* \beta}} C^{* \gamma}+\frac{\overleftarrow{\delta}}{\delta \theta_{\mu}^{\beta}} \theta_{\mu}^{\gamma}\right) \\
& -t_{j k}^{\alpha}\left(\frac{\overleftarrow{\delta}}{\delta \psi_{j}} \psi_{k}+\frac{\overleftarrow{\delta}}{\delta \bar{\psi}_{j}^{*}} \bar{\psi}_{k}^{*}\right) \\
& \left.+\left(\frac{\overleftarrow{\delta}}{\delta \bar{\psi}_{j}} \bar{\psi}_{k}+\frac{\overleftarrow{\delta}_{\delta \psi_{j}^{*}}}{\psi_{k}^{*}}\right) t_{k j}^{\alpha}\right\}
\end{aligned}
$$

We refer to equations of the form (3.15) as the ones of the $T$-symmetry for the corresponding functional.

Using the properties of the functional $\tilde{P}^{(2)}(3.5)$, its locality as well as axial symmetry, Poincare- and $T$ - symmetries we find the general representation,

$$
\begin{aligned}
\tilde{P}^{(2)}= & \int d x\left[Z_{1} \mathcal{A}_{\mu}^{* \alpha} \mathcal{A}_{\mu}^{\alpha}+Z_{2} C^{* \alpha} C^{\alpha}+Z_{3} \psi_{j}^{*} \psi_{j}\right. \\
& \left.+Z_{4} \bar{\psi}_{j}^{*} \bar{\psi}_{j}+Z_{1}^{\prime} \mathcal{A}_{\mu}^{* \alpha} \mathcal{B}_{\mu}^{\alpha}\right],
\end{aligned}
$$

where $Z_{i}, i=1,2,3,4$, " $Z$ ”, " $p$ ” $Z_{1}^{\prime}$ are arbitrary constants. Further, when using the Eq. (3.13) for $\tilde{P}^{(2)}$ we get that $Z_{1}^{\prime}=$ 0 . The final expression for $\tilde{P}^{(2)}$ has the form

$$
\begin{aligned}
\tilde{P}^{(2)}= & \int d x\left[Z_{1} \mathcal{A}_{\mu}^{* \alpha} \mathcal{A}_{\mu}^{\alpha}+Z_{2} C^{* \alpha} C^{\alpha}\right. \\
& \left.+Z_{3} \psi_{j}^{*} \psi_{j}+Z_{4} \bar{\psi}_{j}^{*} \bar{\psi}_{j}\right] .
\end{aligned}
$$

Notice that the functional $\tilde{P}^{(2)}$ does not depend on the fields $\theta_{\mu}^{\alpha}$. By taking (3.18) into account the Eq. (3.12) reduces to the following one

$$
\begin{aligned}
2 \xi \frac{\partial}{\partial \xi} \tilde{P}^{(1)}= & \int d x\left[\left(Z_{1}-1\right)\left(\mathcal{A}_{\mu}^{\alpha} \frac{\delta}{\delta \mathcal{A}_{\mu}^{\alpha}}-\mathcal{A}_{\mu}^{* \alpha} \frac{\delta}{\delta \mathcal{A}_{\mu}^{* \alpha}}\right)\right. \\
& +\left(Z_{2}-1\right)\left(C^{\alpha} \frac{\delta}{\delta C^{\alpha}}-C^{* \alpha} \frac{\delta}{\delta C^{* \alpha}}\right) \\
& +\left(Z_{3}-1\right)\left(\psi_{j} \frac{\delta}{\delta \psi_{j}}-\psi_{j}^{*} \frac{\delta}{\delta \psi_{j}^{*}}\right) \\
& \left.+\left(Z_{4}-1\right)\left(\bar{\psi}_{j} \frac{\delta}{\delta \bar{\psi}_{j}}-\bar{\psi}_{j}^{*} \frac{\delta}{\delta \bar{\psi}_{j}^{*}}\right)\right] \tilde{P}^{(1)},
\end{aligned}
$$

describing the dependence of renormalization constants on the gauge parameter $\xi$. We refer to the Eq. (3.12) as an extended master-equation and to (3.19) as a gauge dependence equation.

\subsection{Solution to the extended master-equation}

Now we consider a solution to the extended master-equation (3.12) for the functional $\tilde{P}^{(1)}$ as presented it in the form

$\tilde{P}^{(1)}=\tilde{P}_{\theta}^{(1)}+\tilde{P}_{\Omega^{*}}^{(1)}+\tilde{P}_{\psi}^{(1)}+\tilde{P}_{\mathcal{A} \mathcal{B}}^{(1)}$

The functional $\tilde{P}_{\theta}^{(1)}$ rewrites as

$\tilde{P}_{\theta}^{(1)}=\int d x \theta_{\mu}^{\alpha}(x) \tilde{P}_{\mu \theta}^{\alpha}(x)$,

and the functionals $\tilde{P}_{\mathcal{A B}}^{(1)}, \tilde{P}_{\psi}^{(1)}, \tilde{P}_{\Omega^{*}}^{(1)}$ do not depend on the fields $\theta_{\mu}^{\alpha}$. By taking into account the properties $\operatorname{dim}\left(\tilde{P}_{\mu \theta}^{\alpha}\right)=$ $2, \operatorname{gh}\left(\tilde{P}_{\mu \theta}^{\alpha}\right)=-1, \varepsilon\left(\tilde{P}_{\mu \theta}^{\alpha}\right)=1, \varepsilon_{f}\left(\tilde{P}_{\mu \theta}^{\alpha}\right)=0$, as well as the Poincare- and $T$-symmetries of the functional $\tilde{P}_{\theta}^{(1)}$, we find that

$$
\begin{aligned}
\tilde{P}_{\mu \theta}^{\alpha} & =-Z_{5} \mathcal{A}_{\mu}^{* \alpha}, \quad \tilde{P}_{\theta}^{(1)}=-Z_{5} \int d x \theta_{\mu}^{\alpha}(x) \mathcal{A}_{\mu}^{* \alpha}(x) \\
& =-Z_{5} \int d x \theta_{\mu}^{\alpha} \mathcal{A}_{\mu}^{* \alpha}
\end{aligned}
$$

where $Z_{5}$ is an arbitrary constant.

The functional $\tilde{P}_{\Omega^{*}}^{(1)}$ is linear in the antifields $\Omega^{*}$ (3.10), and the functionals $\tilde{P}_{\mathcal{A B}}^{(1)}$ and $\tilde{P}_{\psi}^{(1)}$ do not depend on the antifields $\Omega^{*}$. The functional $\tilde{P}_{\Omega^{*}}^{(1)}$ can be represented in the form

$$
\tilde{P}_{\Omega^{*}}^{(1)}=\tilde{P}_{\mathcal{A}^{*}}^{(1)}+\tilde{P}_{C^{*}}^{(1)}+\tilde{P}_{\psi^{*}}^{(1)}+\tilde{P}_{\bar{\psi}^{*}}^{(1)}
$$


By using the arguments analogous to those led us to the structure of the functional $\tilde{P}_{\theta}^{(1)}$ (3.22), we obtain

$$
\begin{aligned}
\tilde{P}_{\mathcal{A}^{*}}^{(1)}= & \int d x\left[Z_{6} \mathcal{A}_{\mu}^{* \alpha} D_{\mu}^{\alpha \beta}(\mathcal{B}) C^{\beta}+g Z_{7 \beta \gamma}^{\alpha} \mathcal{A}_{\mu}^{* \alpha} \mathcal{A}_{\mu}^{\beta} C^{\gamma}\right. \\
& \left.+g Z_{7 \beta \gamma}^{\prime \alpha} \mathcal{A}_{\mu}^{* \alpha} \mathcal{B}_{\mu}^{\beta} C^{\gamma}\right], \\
\tilde{P}_{C^{*}}^{(1)}= & \int d x \frac{g}{2} Z_{8 \beta \gamma}^{\alpha} C^{* \alpha} C^{\beta} C^{\gamma}, \\
\tilde{P}_{\psi^{*}}^{(1)}= & -\int d x g Z_{9 j k}^{\alpha} \psi_{j}^{*} \psi_{k} C^{\alpha}, \\
\tilde{P}_{\bar{\psi}^{*}}^{(1)}= & \int d x g Z_{10 k j}^{\alpha} \bar{\psi}_{j}^{*} \bar{\psi}_{k} C^{\alpha} .
\end{aligned}
$$

Taking into account the gauge symmetry in the external field $\mathcal{B}$ (see the Eq. (3.13)), we find that $Z_{7 \beta \gamma}^{\prime \alpha}=0$. The quantities " $Z$ " introduced in (3.24)-(3.27) are constants that satisfy the equations,

$$
\begin{aligned}
& F_{\gamma \delta}^{\alpha} Z_{7 \delta \sigma}^{\beta}-Z_{7 \gamma \delta}^{\beta} F_{\delta \sigma}^{\alpha}=f^{\alpha \beta \lambda} Z_{7 \gamma \sigma}^{\lambda}, \\
& F_{\gamma \delta}^{\alpha} Z_{8 \delta \sigma}^{\beta}-Z_{8 \gamma \delta}^{\beta} F_{\delta \sigma}^{\alpha}=f^{\alpha \beta \delta} Z_{8 \gamma \sigma}^{\delta}, \\
& t_{j l}^{\alpha} Z_{9 l k}^{\beta}-Z_{9 j l}^{\beta} t_{l k}^{\alpha}=f^{\alpha \beta \gamma} Z_{9 j k}^{\gamma}, \\
& t_{k l}^{\alpha} Z_{10 l j}^{\beta}-Z_{10 k l}^{\beta} t_{l j}^{\alpha}=f^{\alpha \beta \gamma} Z_{10 k j}^{\gamma} .
\end{aligned}
$$

Notice that if $Z_{7 \gamma \sigma}^{\lambda}=Z_{7} f^{\gamma \lambda \sigma}, Z_{8 \gamma \sigma}^{\lambda}=Z_{8} f^{\gamma \lambda \sigma}, Z_{9 j k}^{\alpha}=$ $Z_{9} t_{j k}^{\alpha}, Z_{10 j k}^{\alpha}=Z_{10} t_{j k}^{\alpha}$, then the corresponding Eqs. (3.28)(3.31) hold and the functionals $\tilde{P}_{\mathcal{A}^{*}}^{(1)}, \tilde{P}_{C^{*}}^{(1)}, \tilde{P}_{\psi^{*}}^{(1)}, \tilde{P}_{\bar{\psi}^{*}}^{(1)}(3.24)-$ (3.27) satisfy the Eq. (3.13) by themselves.

In its turn, taking into account the axial symmetry, the Poincare- and the $T$-invariance we determine the general structure of the functional $\tilde{P}_{\psi}^{(1)}$,

$$
\begin{aligned}
\tilde{P}_{\psi}^{(1)}= & \int d x\left[i Z_{11} \bar{\psi}_{j} \gamma^{\mu} D_{\psi \mu}(\mathcal{B}) \psi_{j}\right. \\
& +i g Z_{11 j k}^{\prime \alpha} \bar{\psi}_{j} \gamma^{\mu} \mathcal{B}_{\mu}^{\alpha} \psi_{k}+ \\
& \left.+i g Z_{12 j k}^{\alpha} \bar{\psi}_{j} \gamma^{\mu} \mathcal{A}_{\mu}^{\alpha} \psi_{k}-m Z_{13} \bar{\psi}_{j} \psi_{j}\right],
\end{aligned}
$$

where constants $Z_{12 j k}^{\alpha}$ satisfy the equations

$t_{j l}^{\alpha} Z_{12 l k}^{\beta}-Z_{12 j l}^{\beta} t_{l k}^{\alpha}=f^{\alpha \beta \gamma} Z_{12 j k}^{\gamma}$.

The contribution to the $\tilde{P}_{\psi}^{(1)} \overleftarrow{\tilde{h}^{\alpha}} \omega_{\alpha}$, proportional to $\partial_{\mu} \omega^{\alpha}$, has the form

ig $\bar{\psi}_{j} Z_{11 j k}^{\prime \alpha} \gamma^{\mu} \psi_{k} \partial_{\mu} \omega^{\alpha}$, so that it follows from the Eq. (3.13) that the equalities $Z_{11 j k}^{\prime \alpha}=0$ and

$$
\begin{aligned}
\tilde{P}_{\psi}^{(1)}= & \int d x\left[i Z_{11} \bar{\psi} \gamma^{\mu} D_{\psi \mu}(\mathcal{B}) \psi+i g Z_{12 j k}^{\alpha} \bar{\psi}_{j} \gamma^{\mu} \mathcal{A}_{\mu}^{\alpha} \psi_{k}\right. \\
& \left.-m Z_{13} \bar{\psi}_{j} \psi_{j}\right]
\end{aligned}
$$

hold. Notice that in the case $Z_{12 j k}^{\alpha}=Z_{12} t_{k j}^{\alpha}$ the Eq. (3.33) are fulfilled and the functional $\tilde{P}_{\psi}^{(1)}$ (3.35) satisfies the Eq. (3.13).

Insert the representation for the functional $\tilde{P}^{(1)}$ in the form (3.20) into the Eq. (3.12). Then, analysis of the $\theta \psi \bar{\psi}$ components in the extended master-equation (3.12) yields

$Z_{12 j k}^{\alpha}=Z_{12} t_{j k}^{\alpha}, \quad Z_{12}=Z_{11} / Z_{5}$,

and the possibility to represent the functional $\tilde{P}_{\psi}^{(1)}$ as

$$
\tilde{P}_{\psi}^{(1)}=\int d x\left[i Z_{11} \bar{\psi}_{j} \gamma^{\mu} D_{\psi \mu j k}(U) \psi_{k}-m Z_{13} \bar{\psi}_{j} \psi_{j}\right],
$$

where the notation

$U=\left\{U_{\mu}^{\alpha}\right\}, \quad U_{\mu}^{\alpha}=Z_{5}^{-1} \mathcal{A}_{\mu}^{\alpha}+\mathcal{B}_{\mu}^{\alpha}$

is used. The $\theta \mathcal{A}^{*} C$ components in the Eq. (3.12) lead to the relations

$Z_{7 \beta \gamma}^{\alpha}=Z_{7} f^{\alpha \beta \gamma}, \quad Z_{7}=\frac{Z_{6}}{Z_{5}}$,

and to the representation

$\tilde{P}_{\mathcal{A}^{*}}^{(1)}=\int d x Z_{6} \mathcal{A}_{\mu}^{* \alpha} D_{\mu}^{\alpha \beta}(U) C^{\beta}$.

Consideration of the $\mathcal{A}^{*} \mathcal{A C C}$ components in the Eq. (3.12) gives the relations

$Z_{8 \beta \gamma}^{\alpha}=Z_{8} f^{\alpha \beta \gamma}, \quad Z_{8}=Z_{7}=\frac{Z_{6}}{Z_{5}}$,

and the representation for the functional $\tilde{P}_{C^{*}}^{(1)}$ in the form

$\tilde{P}_{C^{*}}^{(1)}=\int d x \frac{g}{2} \frac{Z_{6}}{Z_{5}} f^{\alpha \beta \gamma} C^{* \alpha} C^{\beta} C^{\gamma}$.

Studying the $\bar{\psi} \psi \partial C, \bar{\psi} \psi \mathcal{B} C$ and $m \bar{\psi} \psi C$ components in the Eq. (3.12) lead to the relations

$Z_{10 j k}^{\alpha}=Z_{9 j k}^{\alpha}, \quad Z_{9 j k}^{\alpha}=Z_{9} t_{j k}^{\alpha}, \quad Z_{9}=\frac{Z_{6}}{Z_{5}}$,

$Z_{10 j k}^{\alpha}=\frac{Z_{6}}{Z_{5}} t_{j k}^{\alpha}$, 
and, as a consequence, to the representation of the functionals $\tilde{P}_{\psi^{*}}^{(1)}$ and $\tilde{P}_{\bar{\psi}}^{(1)}$ as

$$
\begin{aligned}
\tilde{P}_{\psi^{*}}^{(1)} & =-\int d x g \frac{Z_{6}}{Z_{5}} \psi_{j}^{*} t_{j k}^{\alpha} \psi_{k} C^{\alpha}, \\
\tilde{P}_{\bar{\psi}^{*}}^{(1)} & =\int d x g \frac{Z_{6}}{Z_{5}} \bar{\psi}_{j}^{*} t_{k j}^{\alpha} \bar{\psi}_{k} C^{\alpha} \\
& =\int d x g \frac{Z_{6}}{Z_{5}} \bar{\psi}_{j} t_{j k}^{\alpha} \bar{\psi}_{k}^{*} C^{\alpha} .
\end{aligned}
$$

The functional $\tilde{P}_{\mathcal{A B}}^{(1)}$ depends on the fields $\mathcal{A}$ and $\mathcal{B}$ only. The $\theta \mathcal{A B}$ components in the Eq. (3.12) allow us to conclude that the functional $\tilde{P}_{\mathcal{A B}}^{(1)}$ depends on the fields $\mathcal{A}$ and $\mathcal{B}$ only in combination (3.38),

$\tilde{P}_{\mathcal{A B}}^{(1)}(\mathcal{A}, \mathcal{B})=X(U)$.

Finally, consideration of the $\mathcal{A B C}$ components in the Eq. (3.12) leads to equations for the functional $X(U)(3.46)$

$D_{\mu}^{\alpha \beta}(U) \frac{\delta}{\delta U_{\mu}^{\beta}(x)} X(U)=0$.

The required solution to the Eq. (3.47) can be written in the form

$\tilde{P}_{\mathcal{A B}}^{(1)}(\mathcal{A}, \mathcal{B})=X(U)=-\int d x \frac{1}{4} Z_{14} G_{\mu \nu}^{\alpha}(U) G_{\mu \nu}^{\alpha}(U)$,

where

$G_{\mu \nu}^{\alpha}(U)=\partial_{\mu} U_{\nu}^{\alpha}-\partial_{\nu} U_{\mu}^{\alpha}+g f^{\alpha \beta \gamma} U_{\mu}^{\beta} U_{\nu}^{\gamma}$

Thus the general solution to the extended master-equation, $\tilde{P}^{(1)}$, is constructed. It is defined by fifth independent arbitrary constants $Z_{5}, Z_{6}, Z_{11}, Z_{13}, Z_{14}$ and has the form

$$
\begin{aligned}
\tilde{P}^{(1)}= & \int d x\left[-\frac{1}{4} Z_{14} G_{\mu \nu}^{\alpha}(U) G_{\mu \nu}^{\alpha}(U)\right. \\
& +i Z_{11} \bar{\psi}_{j} \gamma^{\mu} D_{\psi \mu j k}(U) \psi_{k}-m Z_{13} \bar{\psi}_{j} \psi_{j} \\
& -Z_{5} \theta_{\mu}^{\alpha} \mathcal{A}_{\mu}^{* \alpha}+Z_{6} \mathcal{A}_{\mu}^{* \alpha} D_{\mu}^{\alpha \beta}(U) C^{\beta} \\
+ & g \frac{Z_{6}}{Z_{5}}\left(f^{\alpha \beta \gamma} C^{* \alpha} C^{\beta} C^{\gamma}+\bar{\psi}_{j}^{*} t_{k j}^{\alpha} \bar{\psi}_{k} C^{\alpha}\right. \\
& \left.\left.-\psi_{j}^{*} t_{j k}^{\alpha} \psi_{k} C^{\alpha}\right)\right] .
\end{aligned}
$$

Notice that at $Z_{1}=Z_{2}=Z_{3}=Z_{4}=Z_{5}=Z_{6}=Z_{11}=$ $Z_{13}=Z_{14}=1$, the equality (the initial condition),

$P_{Z=1}=S_{\text {ext }}$,

holds where $S_{\text {ext }}$ is given by the formula (2.12).
3.2 Solution to the gauge dependence equation

Consider now a solution to the Eq. (3.19) describing the gauge dependence of the constants entering the general solution constructed, $\tilde{P}^{(1)}$, to the extended master-equation (3.50). By studying the $\mathcal{A}^{*} \theta, \mathcal{A} D(U) G(U), \mathcal{A}^{*} f \mathcal{A} C$ and $\bar{\psi} \gamma t \mathcal{A} \psi$ structures in the Eq. (3.19), we derive the following relation

$2 \xi \dot{Z}_{5}=-\left(Z_{1}-1\right) Z_{5} \Rightarrow Z_{1}=1-2 \xi \frac{\dot{Z}_{5}}{Z_{5}}$.

Henceforth we use the notation

$\dot{I} \equiv \frac{\partial}{\partial \xi} I$,

for any quantity $I=I(\xi, \ldots)$ depending on the gauge parameter $\xi$.

Analysis of the $\mathcal{A}^{*} D(U) C$ components in the Eq. (3.19) gives the relation

$2 \xi \dot{Z}_{6}=\left(Z_{2}-Z_{1}\right) Z_{6} \Rightarrow Z_{2}=1+2 \xi\left(\frac{\dot{Z}_{6}}{Z_{6}}-\frac{\dot{Z}_{5}}{Z_{5}}\right)$.

Considering the $\bar{\psi} \gamma D_{\psi}(U) \psi$ components in the Eq. (3.19), we obtain

$2 \xi \dot{Z}_{11}=\left(Z_{3}+Z_{4}-2\right) Z_{11}$.

Analyzing the $m \bar{\psi} \psi$ components in the Eq. (3.19), we find

$2 \xi \dot{Z}_{13}=\left(Z_{3}+Z_{4}-2\right) Z_{13}$.

By making use of the change of constants " $Z$ "

$Z_{13}=Z_{11} Z_{15}, \quad Z_{3}-Z_{4}=2 Z_{16}$,

the Eqs. (3.55), (3.56) rewrite in the form

$\dot{Z}_{15}=0, \quad Z_{3}=1+\xi \frac{\dot{Z}_{11}}{Z_{11}}+Z_{16}, \quad Z_{4}=1+\xi \frac{\dot{Z}_{11}}{Z_{11}}-Z_{16}$

Finally, consideration of the $G(U) G(U)$ components in the Eq. (3.19) leads to the important statement that,

$\dot{Z}_{14}=0$.

Analysis of the $\psi^{*} t \psi C, \bar{\psi}^{*} t^{t} \bar{\psi} C$ and $C^{*} f C C$ components in the Eq. (3.19) gives no new information.

Below, in Sect. 5 find that all constants " $Z$ " can be interpreted as renormalization constants which are uniquely defined from the conditions of divergence elimination.

Let us formulate the results obtained in that Section in the form of a lemma.

Lemma Let

$P=\int d x P\left(Q, Q^{*}, \bar{C}, B, \mathcal{B}, \xi, \theta, \chi\right)$, 
be a local functional of all variables, obey the quantum numbers of the action $S_{\text {ext }}$ and satisfy all Eqs. (2.21)-(2.25) as well as extra symmetries (Poincare-invariance and so on) which have been used in solving the Eqs. (2.21)-(2.25) with substitution $S_{\text {ext }} \rightarrow P$.

Then the functional $P$ has the form

$P=P_{00}+P^{(1)}+\chi P^{(2)}$,

where $P_{00}$ is given by the formula (3.3), $P^{(1)}$ and $P^{(2)}$ do not depend on $B^{\alpha}$ and $\chi$ and are functionals of arguments $\Omega$, $\Omega^{*}, \mathcal{B}, \bar{C}, \xi, \theta$,

$$
\begin{aligned}
P^{(k)} & =P^{(k)}\left(Q, Q^{*}, \bar{C}, \mathcal{B}, \xi, \theta\right)=\tilde{P}^{(k)} \\
& =\tilde{P}^{(k)}\left(\Omega, \Omega^{*}, \mathcal{B}, \bar{C}, \xi, \theta\right), k=1,2, \\
\Omega & =\{\mathcal{A}, \psi, \bar{\psi}, C\}, \Omega^{*}=\left\{\mathcal{A}^{*}, \psi^{*}, \bar{\psi}^{*}, C^{*}\right\}, \\
\mathcal{A}_{\mu}^{* \alpha} & =A_{\mu}^{* \alpha}-D_{\mu}^{\alpha \beta}(\mathcal{B}) \bar{C}^{\beta}, \mathcal{A}_{\mu}^{\alpha}=A_{\mu}^{\alpha} .
\end{aligned}
$$

The functionals $\tilde{P}^{(k)}$ read

$$
\begin{aligned}
\tilde{P}^{(1)}= & \int d x\left[-\frac{1}{4} Z_{14} G_{\mu \nu}^{\alpha}(U) G_{\mu \nu}^{\alpha}(U)\right. \\
& +i Z_{11} \bar{\psi}_{j} \gamma^{\mu} D_{\psi \mu j k}(U) \psi_{k}-m Z_{13} \bar{\psi}_{j} \psi_{j} \\
& -Z_{5} \theta_{\mu}^{\alpha} \mathcal{A}_{\mu}^{* \alpha}++Z_{6} \mathcal{A}_{\mu}^{* \alpha} D_{\mu}^{\alpha \beta}(U) C^{\beta} \\
+ & g \frac{Z_{6}}{Z_{5}}\left(f^{\alpha \beta \gamma} C^{* \alpha} C^{\beta} C^{\gamma}+\bar{\psi}_{j}^{*} t_{k j}^{\alpha} \bar{\psi}_{k} C^{\alpha}\right. \\
& \left.\left.-\psi_{j}^{*} t_{j k}^{\alpha} \psi_{k} C^{\alpha}\right)\right], \\
\tilde{P}^{(2)}= & \int d x\left[Z_{1} \mathcal{A}_{\mu}^{* \alpha} \mathcal{A}_{\mu}^{\alpha}+Z_{2} C^{* \alpha} C^{\alpha}+Z_{3} \psi_{j}^{*} \psi_{j}\right. \\
& \left.+Z_{4} \bar{\psi}_{j}^{*} \bar{\psi}_{j}\right] . \\
U=\{ & \left.U_{\mu}^{\alpha}\right\}, \quad U_{\mu}^{\alpha}=Z_{5}^{-1} \mathcal{A}_{\mu}^{\alpha}+\mathcal{B}_{\mu}^{\alpha}, \\
Z_{1}=1 & -2 \xi \frac{Z_{5}}{Z_{5}}, \quad Z_{2}=1+2 \xi\left(\frac{\dot{Z}_{6}}{Z_{6}}-\frac{\dot{Z}_{5}}{Z_{5}}\right), \\
Z_{3}=1 & +\xi \frac{\dot{Z}_{11}}{Z_{11}}+Z_{16}, \quad Z_{4}=1+\xi \frac{\dot{Z}_{11}}{Z_{11}}-Z_{16}, \\
Z_{13}= & Z_{11} Z_{15}, \quad \dot{Z}_{14}=0, \quad \dot{Z}_{15}=0,
\end{aligned}
$$

where $Z_{5}, Z_{6}, Z_{11}, Z_{16}$ are arbitrary constants depending perhaps on $\xi$, and $Z_{14}, Z_{15}$ are arbitrary constants not depending on $\xi$.

The inverse statement, being perhaps trivial but nevertheless important, is true: if the functional $P$ has the form (3.61), (3.62), (3.65), (3.66) and the relations (3.67), (3.68), (3.69) are fulfilled then this functional satisfies the Eqs. (2.21)(2.25).

\section{Generating functional of vertex functions}

It is convenient to define the generating functional of Green functions by making use of the action functional $P$ constructed in the previous Section as the action yields then a finite theory certainly. In what follows we re-denote the functional $P, P \equiv S_{R}$, and, respectively, $P^{(k)} \equiv S_{R}^{(k)}$, $\tilde{P}^{(k)} \equiv \tilde{S}_{R}^{(k)}, k=1,2$.

The generating functional of Green functions is given by the functional integral,

$$
\begin{aligned}
Z\left(J_{\Phi}, L\right) & =\int d \Phi \exp \left(\frac{i}{\eta}\left[S_{R}+J_{\Phi} \Phi\right]\right) \\
& =\exp \left\{\frac{i}{\eta} W\left(J_{\Phi}, L\right)\right\},
\end{aligned}
$$

with $\eta$ standing for a parameter of a loop expansion as to the expression in the exponential in $(4.1), W\left(J_{\Phi}, L\right)$ that is the generating functional of connected Green functions, and the notations are introduced $\Phi=\{Q, \bar{C}, B\}$ " $Z$ ", " $p$ " $L=\left\{L^{A}\right\}=\left\{\mathcal{B}, Q^{*}, \xi, \theta, \chi\right\}$, and $J_{\Phi}$ as for the sources to the fields $\Phi$. Also, we assume that all the constants " $Z$ " are functions of $\eta$, " $Z$ " $=$ " $Z$ " $(\eta)$, expandable in Taylor power series, $Z_{i}(0)=1, \dot{Z}_{i}=O(\eta), i=5,6,11,14,15, Z_{16}=$ $O(\eta)$. In that case the functional $S_{R}$ becomes a function of $\eta$,

$S_{R}=S_{R}(\eta)=\sum_{l=0}^{\infty} \eta^{l} S_{R, l}, \quad S_{R}^{[k]}=\sum_{l=0}^{k} \eta^{l} S_{R, l}$,

so that all the functionals $S_{R, l}$ are linear combination of a single set of monomials,

$S_{R, l}=\sum_{i=1}^{I} a_{l, i} S_{i}$,

where $\left\{S_{i}, i=1, \ldots, I\right\}$ is a sub-set of monomials which the action $S_{\text {ext }}$ is expanded in, and $a_{l, i}$ are constant coefficients for $l$-loop order.

The generating functional of vertex Green functions (effective action) is defined by the Legendre transformation

$\Gamma\left(\Phi_{m \mid}, L\right)=W\left(J_{\Phi}, L\right)-J_{\Phi} \Phi_{m \mid}, \quad \Phi_{m \mid}=\frac{\delta}{\delta J_{\Phi}} W\left(J_{\Phi}, L\right)$,

has the quantum numbers $\varepsilon(\Gamma)=0, \operatorname{gh}(\Gamma)=0, \operatorname{dim}(\Gamma)=$ $0, \varepsilon_{f}(\Gamma)=0$, and satisfies the relations

$$
\begin{gathered}
\Gamma\left(\Phi_{m \mid}, L\right) \frac{\overleftarrow{\delta}}{\delta \Phi_{m \mid}}=-J_{\Phi}\left(\Phi_{m \mid}, L\right) \\
\Gamma\left(\Phi_{m \mid}, L\right) \frac{\overleftarrow{\delta}}{\delta L^{A}}=W\left(J_{\Phi}, L\right) \frac{\overleftarrow{\delta}}{\delta L^{A}}
\end{gathered}
$$


Functional average of the Eqs. (2.21)-(2.25) with substitution $S_{\text {ext }} \rightarrow S_{R}$ yields the corresponding equations for the functional $\Gamma=\Gamma\left(\Phi_{m \mid}, L\right)$, copying the equations for $S_{R}$,

$$
\begin{aligned}
& \int d x\left(\Gamma \frac{\overleftarrow{\delta}}{\delta Q_{m \mid}} \frac{\delta}{\delta Q^{*}} \Gamma-B_{m \mid} \frac{\delta}{\delta \bar{C}_{m \mid}} \Gamma-\theta \frac{\delta}{\delta \mathcal{B}} \Gamma\right) \\
& +2 \chi \xi \frac{\partial}{\partial \xi} \Gamma+\chi \int d x\left[\left(Q_{m \mid} \frac{\delta}{\delta Q_{m \mid}}-Q^{*} \frac{\delta}{\delta Q^{*}}\right.\right. \\
& \left.\left.-\bar{C}_{m \mid} \frac{\delta}{\delta \bar{C}_{m \mid}}-B_{m \mid} \frac{\delta}{\delta B_{m \mid}}\right) \Gamma\right]=0, \\
& \Gamma \overleftarrow{H_{m \mid}^{\alpha}} \omega_{\alpha}=0,
\end{aligned}
$$

where $\overleftarrow{H_{m \mid}^{\alpha}} \omega_{\alpha}$ is given by the expression (2.23) with the replacement $\Phi \rightarrow \Phi_{m \mid}$,

$$
\begin{aligned}
& \frac{\delta}{\delta B_{m \mid}^{\alpha}} \Gamma=D_{\mu}^{\alpha \beta}(\mathcal{B}) A_{m \mid \mu}^{\beta}+\xi B_{m \mid}^{\alpha}, \\
& D_{\mu}^{\alpha \beta}(\mathcal{B}) \frac{\delta}{\delta A_{\mu}^{* \beta}} \Gamma-\frac{\delta}{\delta \bar{C}_{m \mid}^{\alpha}} \Gamma=-g f^{\alpha \beta \gamma} A_{m \mid \mu}^{\beta} \theta_{\mu}^{\gamma}
\end{aligned}
$$

Represent the functional $\Gamma$ in the following form

$\Gamma=\Gamma_{00}+\Gamma^{(1)}+\chi \Gamma^{(2)}$,

where

$\Gamma_{00}=\int d x\left(B_{m \mid} D(\mathcal{B}) A_{m \mid}+\frac{\xi}{2} B_{m \mid}^{2}+g \theta_{\mu}^{\alpha} f^{\alpha \beta \gamma} A_{m \mid \mu}^{\beta} \bar{C}_{m \mid}^{\gamma}\right)$,

and the functionals $\Gamma^{(1)}$ and $\Gamma^{(2)}$ do not depend on the parameter $\chi$. Due to the structure chosen for the functional (4.11) it follows from the Eqs. (4.8) and (4.9) that the functionals $\Gamma^{(1)}$ and $\Gamma^{(2)}$ do not depend on the fields $B_{m l}^{\alpha}$,

$$
\begin{aligned}
\frac{\delta}{\delta B_{m} \mid} \Gamma^{(k)} & =0, \Gamma^{(k)}=\Gamma^{(k)}\left(Q_{m \mid}, \bar{C}_{m \mid}, \mathcal{B}, Q^{*}, \xi, \theta\right), \\
k & =1,2,
\end{aligned}
$$

and satisfy the equations

$$
\left(D_{\mu}^{\alpha \beta}(\mathcal{B}) \frac{\delta}{\delta A_{\mu}^{* \beta}}-\frac{\delta}{\delta \bar{C}_{m \mid}^{\alpha}}\right) \Gamma^{(k)}=0, \quad k=1,2,
$$

In its turn, the Eq. (4.6) splits in the two, one of which is closed as for the functional $\Gamma^{(1)}$,

$$
\begin{aligned}
& \int d x {\left[\Gamma^{(1)} \frac{\overleftarrow{\delta}}{\delta Q_{m \mid}} \frac{\delta}{\delta Q^{*}} \Gamma^{(1)}-g \theta_{\mu}^{\alpha} f^{\alpha \beta \gamma} \bar{C}_{m \mid}^{\beta} \frac{\delta}{\delta A_{\mu}^{* \gamma}} \Gamma^{(1)}\right.} \\
&\left.-\theta_{\mu}^{\alpha} \frac{\delta}{\delta \mathcal{B}_{\mu}^{\alpha}} \Gamma^{(1)}\right]=0
\end{aligned}
$$

and the second includes both the functionals and describes their dependence on the gauge parameter $\xi$,

$$
\begin{aligned}
2 \xi \frac{\partial}{\partial \xi} \Gamma^{(1)}= & \int d x\left[\Gamma^{(1)}\left(\frac{\overleftarrow{\delta}}{\delta Q_{m \mid}} \frac{\delta}{\delta Q^{*}}-\frac{\overleftarrow{\delta}}{\delta Q^{*}} \frac{\delta}{\delta Q_{m \mid}}\right) \Gamma^{(2)}\right. \\
& \left.-\left(g \theta_{\mu}^{\alpha} f^{\alpha \beta \gamma} \bar{C}_{m \mid}^{\beta} \frac{\delta}{\delta A_{\mu}^{* \gamma}}+\theta_{\mu}^{\alpha} \frac{\delta}{\delta \mathcal{B}_{\mu}^{\alpha}}\right) \Gamma^{(2)}\right] \\
& +\int d x\left[\left(\bar{C}_{m \mid} \frac{\delta}{\delta \bar{C}_{m \mid}}-Q_{m \mid} \frac{\delta}{\delta Q_{m}}\right.\right. \\
& \left.\left.+Q^{*} \frac{\delta}{\delta Q^{*}}\right) \Gamma^{(1)}\right]
\end{aligned}
$$

The Eq. (4.7) rewrites now in the form of the two equations as for the functionals $\Gamma^{(1)}$ and $\Gamma^{(2)}$,

$\Gamma^{(k)} \overleftarrow{h_{m \mid}^{\alpha}} \omega_{\alpha}=0, \quad k=1,2$

where

$$
\begin{aligned}
\overleftarrow{h_{m \mid}^{\alpha}} \omega_{\alpha}= & \int d x\left\{\left[\frac{\overleftarrow{\delta}}{\delta \mathcal{B}_{\mu}^{\beta}} D_{\mu}^{\beta \alpha}(\mathcal{B})\right.\right. \\
& +g f^{\beta \gamma \alpha}\left(\frac{\overleftarrow{\delta}}{\delta A_{m \mid \mu}^{\beta}} A_{m \mid \mu}^{\gamma}+\frac{\overleftarrow{\delta}}{\delta C_{m \mid}^{\beta}} C_{m \mid}^{\gamma}\right) \\
& +g f^{\beta \gamma \alpha}\left(\frac{\overleftarrow{\delta}}{\delta \bar{C}_{m \mid}^{\beta}} \bar{C}_{m \mid}^{\gamma}+\frac{\overleftarrow{\delta}}{\delta A_{\mu}^{* \beta}} A_{\mu}^{* \gamma}+\frac{\overleftarrow{\delta}}{\delta C^{* \beta}} C^{* \gamma}+\frac{\overleftarrow{\delta}}{\delta \theta_{\mu}^{\beta}} \theta_{\mu}^{\gamma}\right) \\
& -g t_{j k}^{\alpha}\left(\frac{\overleftarrow{\delta}}{\delta \psi_{m \mid j}} \psi_{m \mid k}+\frac{\overleftarrow{\delta}}{\delta \bar{\psi}_{j}^{*}} \bar{\psi}_{k}^{*}\right) \\
& \left.\left.+g\left(\frac{\overleftarrow{\delta}}{\delta \bar{\psi}_{m \mid j}} \bar{\psi}_{m \mid k}+\frac{\overleftarrow{\delta}}{\delta \psi_{j}^{*}} \psi_{k}^{*}\right) t_{k j}^{\alpha}\right] \omega_{\alpha}\right\} .
\end{aligned}
$$

As for the Eq. (4.13), it is convenient to introduce the variables $\mathcal{A}_{\mu}^{* \alpha}=\mathcal{A}_{\mu}^{* \alpha}(x), \mathcal{A}_{m \mid \mu}^{* \alpha}=\mathcal{A}_{m \mid \mu}^{* \alpha}(x)$

$\mathcal{A}_{\mu}^{* \alpha}=A_{\mu}^{* \alpha}-D_{\mu}^{\alpha \beta}(\mathcal{B}) \bar{C}^{\beta}, \quad \mathcal{A}_{m \mid \mu}^{* \alpha}=A_{\mu}^{* \alpha}-D_{\mu}^{\alpha \beta}(\mathcal{B}) \bar{C}_{m \mid}^{\beta}$,

and to use the following convention

$\mathcal{A}_{\mu}^{\alpha}=A_{\mu}^{\alpha}$,

as for the sake of uniformity. Also, introduce the new functionals $\tilde{\Gamma}^{(k)}$ by the rule,

$$
\begin{aligned}
& \tilde{\Gamma}^{(k)}\left(\mathcal{B}, \bar{C}_{m \mid}, \mathcal{A}_{m \mid}^{*}, \Lambda_{m \mid}\right) \\
& \quad=\left.\Gamma^{(k)}\left(\mathcal{B}, \bar{C}_{m \mid}, A^{*}, \Lambda_{m \mid}\right)\right|_{A^{*} \rightarrow \mathcal{A}_{m \mid}^{*}+D_{\mu}^{\alpha \beta}(\mathcal{B}) \bar{C}_{m \mid}^{\beta},}
\end{aligned}
$$

where the notation

$$
\begin{aligned}
& \Lambda=\left\{Q, \psi^{*}, \bar{\psi}^{*}, C^{*}, \xi, \theta\right\}, \\
& \Lambda_{m \mid}=\left\{Q_{m \mid}, \psi^{*}, \bar{\psi}^{*}, C^{*}, \xi, \theta\right\}
\end{aligned}
$$


is used. With the definitions (4.18)-(4.20) taken into account, we have

$$
\begin{aligned}
& \frac{\delta}{\delta A_{\mu}^{* \alpha}} \Gamma^{(k)}=\frac{\delta}{\delta \mathcal{A}_{m \mid \mu}^{* \alpha}} \tilde{\Gamma}^{(k)}, \\
& \frac{\delta}{\delta \bar{C}_{m \mid}^{\alpha}} \Gamma^{(k)}= \frac{\delta}{\delta \bar{C}_{m \mid}^{\alpha}} \tilde{\Gamma}^{(k)}+D_{\mu}^{\alpha \beta}(\mathcal{B}) \frac{\delta}{\delta \mathcal{A}_{m \mid \mu}^{* \beta}} \tilde{\Gamma}^{(k)}, \\
& \frac{\delta}{\delta \mathcal{B}_{\mu}^{\alpha}} \Gamma^{(k)}= \frac{\delta}{\delta \mathcal{B}_{\mu}^{\alpha}} \tilde{\Gamma}^{(k)}-g f^{\alpha \beta \gamma} \bar{C}_{m \mid}^{\beta} \frac{\delta}{\delta \mathcal{A}_{m \mid \mu}^{* \gamma}} \tilde{\Gamma}^{(k)}, \\
& k=1,2 .
\end{aligned}
$$

Then, we find from the Eqs. (4.13), (4.20), (4.22), (4.23) that

$\frac{\delta}{\delta \bar{C}_{m \mid}^{\alpha}} \tilde{\Gamma}^{(k)}=0$,

the functionals $\tilde{\Gamma}^{(k)}, k=1,2$, do not depend on the fields $\bar{C}_{m \mid}^{\alpha}$,

$\tilde{\Gamma}^{(k)}=\tilde{\Gamma}^{(k)}\left(\Omega_{m \mid}, \Omega_{m \mid}^{*}, \mathcal{B}, \xi, \theta\right)$.

Henceforth we use the notations

$\Omega_{m \mid}=\left\{\mathcal{A}_{m \mid}, \psi_{m \mid}, \bar{\psi}_{m \mid}, C_{m \mid}\right\}, \quad \mathcal{A}=A$,

$\Omega_{m \mid}^{*}=\left\{\mathcal{A}_{m \mid}^{*}, \psi^{*}, \bar{\psi}^{*}, C^{*}\right\}, \quad k=1,2$.

Now, with (4.20), (4.26), (4.27) taken into account, the ones (4.14), (4.15) rewrite in the form

$$
\begin{aligned}
& \frac{1}{2}\left(\tilde{\Gamma}^{(1)}, \tilde{\Gamma}^{(1)}\right)-\int d x\left(\theta \frac{\delta}{\delta \mathcal{B}}\right) \tilde{\Gamma}^{(1)}=0, \\
2 \xi \frac{\partial}{\partial \xi} \tilde{\Gamma}^{(1)}=\left(\tilde{\Gamma}^{(1)}, \tilde{\Gamma}^{(2)}\right) & \\
+ & \int d x\left(\Omega_{m \mid}^{*} \frac{\delta}{\delta \Omega_{m \mid}^{*}}-\Omega_{m \mid} \frac{\delta}{\delta \Omega_{m \mid}}\right) \tilde{\Gamma}^{(1)} \\
- & \int d x\left(\theta \frac{\delta}{\delta \mathcal{B}}\right) \tilde{\Gamma}^{(2)},
\end{aligned}
$$

where the notation for the antibracket $[8,9]$ is used,

$$
(F, G)=\frac{1}{2} F \int d x\left(\frac{\overleftarrow{\delta}}{\delta \Omega_{m \mid}} \frac{\delta}{\delta \Omega_{m \mid}^{*}}-\frac{\overleftarrow{\delta}}{\delta \Omega_{m \mid}^{*}} \frac{\delta}{\delta \Omega_{m \mid}}\right) G
$$

Further, with the relations (4.20), (4.26) and

$$
\begin{aligned}
\Gamma^{(k)} & \int d x\left[\frac{\overleftarrow{\delta}}{\delta \mathcal{B}_{\mu}^{\beta}} D_{\mu}^{\beta \alpha}(\mathcal{B})\right. \\
& \left.+g f^{\beta \gamma \alpha}\left(\frac{\overleftarrow{\delta}}{\delta \bar{C}_{m \mid}^{\beta}} \bar{C}_{m \mid}^{\gamma}+\frac{\overleftarrow{\delta}}{\delta A_{\mu}^{* \beta}} A_{\mu}^{* \gamma}\right)\right] \\
& =\tilde{\Gamma}^{(k)} \int d x\left[\frac{\overleftarrow{\delta}}{\delta \mathcal{B}_{\mu}^{\beta}} D_{\mu}^{\beta \alpha}(\mathcal{B})+g f^{\beta \gamma \alpha} \frac{\overleftarrow{\delta}}{\delta \mathcal{A}_{m \mid \mu}^{* \beta}} \mathcal{A}_{m \mid \mu}^{* \gamma}\right]
\end{aligned}
$$

we find that

$\Gamma^{(k)} \overleftarrow{h_{m \mid}^{\alpha}} \omega_{\alpha}=\tilde{\Gamma}^{(k)} \overleftarrow{\tilde{h}_{m \mid}^{\alpha}} \omega_{\alpha}=0, \quad k=1,2$

where the operator $\overleftarrow{\overleftarrow{h}_{m \mid}^{\alpha}} \omega_{\alpha}$ is defined in the equality (3.14) with the replacement $\Omega \rightarrow \Omega_{m \mid}, \Omega^{*} \rightarrow \Omega_{m \mid}^{*}$.

Then, when studying the tensor structure of divergence parts of the generating functional of vertexes, it is convenient to use a consequence of the Eq. (4.32) in particular case $\omega_{\alpha}(x)=$ const, i.e. as to a global $\left.T_{m}\right|^{\text {-symmetry: }}$

$\tilde{\Gamma}^{(k)} \overleftarrow{T_{m \mid}^{\alpha}}=0, k=1,2$

where the operators $\overleftarrow{T_{m}^{\alpha}}$ are defined by the Eq. (3.16) with the replacement $\Omega \rightarrow \Omega_{m \mid}, \Omega^{*} \rightarrow \Omega_{m \mid}^{*}$.

\section{Renormalization}

In that section we study the structure of renormalizations, and show the multiplicative character of the renormalizabiliuty of the model considered. The main role in that study is given to resolving the extended master-equation (3.12) and the one (3.19) describing the gauge dependence. We show that the renormalized quantum action and the effective action satisfy exactly their master equations to each subsequent order in loops. In this resolving, the structure of the renormalized quantum action is determined by the same monomials in fields and antifields as it does for the non-renormalized quantum action with constants determined by the divergencies of the effective action. For the sake of notational simplicity, we omit lower case $m \mid$ of any arguments of any functionals.

\subsection{Tree approximation $(\eta=0)$}

Consider the tree approximation for the functional $\Gamma, \Gamma_{0}=$ $S_{\text {ext }}$, written in new variables as

$$
\begin{aligned}
\Gamma_{0} & =\Gamma_{00}+\Gamma_{0}^{(1)}+\chi \Gamma_{0}^{(2)}, \\
\Gamma_{0}^{(1)} & =\tilde{\Gamma}_{0}^{(1)}, \quad \Gamma_{0}^{(2)}=\tilde{\Gamma}_{0}^{(2)},
\end{aligned}
$$

where

$$
\Gamma_{00}=\int d x\left(B^{\alpha} D_{\mu}^{\alpha \beta}(\mathcal{B}) A_{\mu}^{\beta}+\frac{\xi}{2} B^{\alpha} B^{\alpha}+g \theta_{\mu}^{\alpha} f^{\alpha \beta \gamma} A_{\mu}^{\beta} \bar{C}^{\gamma}\right) .
$$

Represent the functional $\tilde{\Gamma}_{0}^{(1)}$ in the form

$$
\begin{aligned}
\tilde{\Gamma}_{0}^{(1)}= & \Gamma_{0 \theta}+\Gamma_{0 \Omega^{*}}+\Gamma_{0 \psi}+\Gamma_{0 \mathcal{A B}}, \\
\Gamma_{0 \psi}= & \Gamma_{0 \psi \mid 1}+\Gamma_{0 \psi \mid 2}, \quad \Gamma_{0 \Omega^{*}}=\Gamma_{0 \mathcal{A}^{*}} \\
& +\Gamma_{0 C^{*}}+\Gamma_{0 \psi^{*}}+\Gamma_{0 \bar{\psi}^{*}},
\end{aligned}
$$


where the following notations being further useful

$$
\begin{aligned}
\Gamma_{0 \theta} & =\int d x \mathcal{A}_{\mu}^{* \alpha} \theta_{\mu}^{\alpha}, \\
\Gamma_{0 \mathcal{A}^{*}} & =\int d x \mathcal{A}_{\mu}^{* \alpha} D_{\mu}^{\alpha \beta}(\mathcal{A}+\mathcal{B}) C^{\beta}, \\
\Gamma_{0 C^{*}} & =\int d x \frac{g}{2} f^{\alpha \beta \gamma} C^{* \alpha} C^{\beta} C^{\gamma}, \\
\Gamma_{0 \psi^{*}} & =-\int d x g \psi_{j}^{*} t_{j k}^{\alpha} \psi_{k} C^{\alpha}, \\
\Gamma_{0 \bar{\psi}^{*}} & =\int d x g \bar{\psi}_{j}^{*} t_{k j}^{\alpha} \bar{\psi}_{k} C^{\alpha}, \\
\Gamma_{0 \psi \mid 1} & =\int d x\left[i \bar{\psi} \gamma^{\mu} D_{\psi \mu}(\mathcal{A}+\mathcal{B}) \psi\right], \\
\Gamma_{0 \psi \mid 2} & =-m \int d x \bar{\psi} \psi, \\
\Gamma_{0 \mathcal{A B}} & =-\int d x \frac{1}{4} G_{\mu \nu}^{\alpha}(\mathcal{A}+\mathcal{B}) G_{\mu \nu}^{\alpha}(\mathcal{A}+\mathcal{B}),
\end{aligned}
$$

are introduced. In its turn, the functional $\tilde{\Gamma}_{0}^{(2)}$ has the form

$$
\tilde{\Gamma}_{0}^{(2)}=\int d x\left[\mathcal{A}_{\mu}^{* \alpha} \mathcal{A}_{\mu}^{\alpha}+C^{* \alpha} C^{\alpha}+\psi_{j}^{*} \psi_{j}+\bar{\psi}_{j}^{*} \bar{\psi}_{j}\right] .
$$

Remind that the functional $\Gamma_{0}$ satisfies the Eqs. (2.21)-(2.25).

\section{$5.2(1+1)$-loop approximation}

We carry out the proof of the multiplicative renormalizability via the mathematical induction method in the framework of loop expansion of the effective action with the use of the minimal subtraction scheme. To this end we suppose that we managed to find such parameters $Z_{i}^{[l]}$,

$$
\begin{aligned}
& Z_{i}^{[l]}= \sum_{n=0}^{l} \eta^{n} z_{i, n}, \quad i=5,6,11,14,15,16, \\
& \dot{Z}_{14}^{[l]}=\dot{Z}_{15}^{[l]}=0, \\
& 1-2 \xi \frac{\dot{Z}_{5}^{[l]}}{Z_{5}^{[l]}}=Z_{1}^{[l]}+O\left(\eta^{l+1}\right), \\
& 1+2 \xi\left(\frac{\dot{Z}_{6}^{[l]}}{Z_{6}^{[l]}}-\frac{\dot{Z}_{5}^{[l]}}{Z_{5}^{[l]}}\right)=Z_{2}^{[l]}+O\left(\eta^{l+1}\right), \\
& 1+\xi \frac{\dot{Z}_{11}^{[l]}}{Z_{11}^{[l]}}+Z_{16}^{[l]}=Z_{3}^{[l]}+O\left(\eta^{l+1}\right), \\
& 1+\xi \frac{\dot{Z}_{11}^{[l]}}{Z_{11}^{[l]}}-Z_{16}^{[l]}=Z_{4}^{[l]}+O\left(\eta^{l+1}\right),
\end{aligned}
$$

that the $l$-loop approximation for $\Gamma, \Gamma^{[l]}=\sum_{n=0}^{l} \eta^{n} \Gamma_{n}$, is a finite functional. We are to show that it is possible to pick up the $l+1$-loop approximation for $Z_{i}$,

$$
\begin{aligned}
& Z_{i}=Z_{i}^{[l]}+z_{i, l+1}+O\left(\eta^{l+2}\right), \quad i=5,6,11,14,15,16, \\
& \dot{z}_{14, l+1}=\dot{z}_{15, l+1}=0,
\end{aligned}
$$

which does compensate the divergences of $l+1$-loop approximation for the functional $\Gamma$.

Represent the action $S_{R}$ in the form

$S_{R}=S_{R}^{[l]}+\eta^{l+1} s_{l+1}+O\left(\eta^{l+2}\right)$,

where $S_{R}^{[l]}$ is the action $S_{R}$ with independent parameters $Z_{i}$ replaced by $Z_{i}^{[l]}$, and satisfying the Eqs. (2.21)-(2.25), and the functional $s_{l+1}$ reads

$s_{l+1}=s_{l+1}^{(1)}+\chi s_{l+1}^{(2)}$.

For the functional $s_{l+1}^{(1)}$ we use the representation

$s_{l+1}^{(1)}=s_{\theta, l+1}+s_{\Omega^{*}, l+1}+s_{\psi, l+1}+s_{\mathcal{A B}, l+1}$,

where

$$
\begin{aligned}
s_{\theta, l+1}= & z_{5, l+1} \Gamma_{0 \theta}, \\
s_{\mathcal{A}^{*}, l+1}= & z_{6, l+1} \Gamma_{0 \mathcal{A}^{*}}-z_{5, l+1} \mathcal{A} \partial_{\mathcal{A}} \Gamma_{0 \mathcal{A}^{*}}, \\
s_{C^{*}, l+1}= & \left(z_{6, l+1}-z_{5, l+1}\right) \Gamma_{0 C^{*}}, \\
s_{\psi^{*}, l+1}= & \left(z_{6, l+1}-z_{5, l+1}\right) \Gamma_{0 \psi^{*}}, \\
s_{\bar{\psi}^{*}, l+1}= & \left(z_{6, l+1}-z_{5, l+1}\right) \Gamma_{0 \bar{\psi}^{*}}, \\
s_{\psi, l+1}= & z_{11, l+1} \Gamma_{0 \psi \mid 1}-z_{5, l+1} \mathcal{A} \partial_{\mathcal{A}} \Gamma_{0 \psi \mid 1} \\
& +\left(z_{11, l+1}+z_{15, l+1}\right) \Gamma_{0 \psi \mid 2}, \\
s_{\mathcal{A B}, l+1}= & z_{14, l+1} \Gamma_{0 \mathcal{A B}}-z_{5, l+1} \mathcal{A} \partial_{\mathcal{A}} \Gamma_{0 \mathcal{A B}} .
\end{aligned}
$$

In its turn, the functional $s_{l+1}^{(2)}$ has the form

$$
\begin{aligned}
s_{l+1}^{(2)}= & \int d x\left[2 \xi \dot{z}_{5, l+1} \mathcal{A}^{*} \mathcal{A}+2 \xi\left(\dot{z}_{6, l+1}-\dot{z}_{5, l+1}\right) C^{*} C\right. \\
& +\xi\left(\dot{z}_{11, l+1}+z_{16, l+1}\right) \psi^{*} \psi+ \\
& \left.+\xi\left(\dot{z}_{11, l+1}-z_{16, l+1}\right) \bar{\psi}^{*} \bar{\psi}\right] .
\end{aligned}
$$

Here (and below in this section) we use the abbreviation to denote the variational derivatives of the kind

$$
\frac{\delta}{\delta \mathcal{A}} \rightarrow \partial_{\mathcal{A}}, \quad \mathcal{A}_{\mathcal{A}}=\int d x \mathcal{A} \frac{\delta}{\delta \mathcal{A}},
$$

when it does not cause an ambiguity.

Let us study the structure of the functional $\Gamma$ with the accuracy including the $(l+1)$-loop approximation. It is described by the diagrams with vertexes from the action $S_{R}$ with parameters $z_{i, n}, i=5,6,11,14,15,16,0 \leq n \leq l+1$, or, in other words, by vertexes from the action $S_{R}^{[l]}$ and from the summand $s_{l+1}$. As we are interested in diagrams of the loop order not higher than $l+1$, the vertexes from $s_{l+1}$ cannot appear in loop diagrams, i.e. vertexes from $s_{l+1}$ give the "tree" contribution to $\Gamma$, equal to $\eta^{l+1} s_{m \mid, l+1}$. Other diagrams are generated by 
the action $S_{R}^{[l]}$. Let $\Gamma\left(S_{R}^{[l]}\right)$ be the contribution of those diagrams into the functional $\Gamma$, i.e.

$\Gamma=\Gamma\left(S_{R}^{[l]}\right)+\eta^{l+1} s_{m \mid, l+1}+O\left(\eta^{l+2}\right)$.

As the functional $S_{R}^{[l]}$ satisfies the Eqs. (2.12)-(2.15), the functional $\Gamma\left(S_{R}^{[l]}\right)$ satisfies the same equations with the replacement $Q, \bar{C}, B \rightarrow Q_{m \mid}, \bar{C}_{m \mid}, B_{m \mid}$.

Represent the functional $\Gamma\left(S_{R}^{[l]}\right)$ in the form

$\Gamma\left(S_{R}^{[l]}\right)=\Gamma_{00}+\Gamma^{(1)}\left(S_{R}^{[l]}\right)+\chi \Gamma^{(2)}\left(S_{R}^{[l]}\right)$,

By repeating the calculations of Sect. 3 we find that

$$
\begin{gathered}
\Gamma^{(k)}\left(S_{R}^{[l]} \mid Q_{m \mid}, \bar{C}_{m \mid}, \mathcal{B}, Q^{*}, B_{m \mid}, \xi, \theta\right)=\tilde{\Gamma}^{(k)} \\
\left(S_{R}^{[l]} \mid \Omega_{m \mid}, \Omega_{m \mid}^{*}, \mathcal{B}, \xi, \theta\right), k=1,2,
\end{gathered}
$$

and the functionals $\tilde{\Gamma}^{(k)}\left(S_{R}^{[l]}\right)$ satisfy the Eqs. (4.28), (4.29) and (4.32).

Represent the functionals $\tilde{\Gamma}^{(k)}\left(S_{R}^{[l]}\right)$ in the form of sums of divergent and finite (after removing a regularization) parts. Taking into account that the functionals $\tilde{\Gamma}^{(k)}\left(S_{R}^{[l]}\right)$ are, by assumption, finite to the $n$-loop approximations, $0 \leq n \leq l$, we obtain

$$
\begin{aligned}
\tilde{\Gamma}^{(k)}\left(S_{R}^{[l]}\right)= & \tilde{\Gamma}^{(k)}\left(S_{R}^{[l]}\right)_{\mathrm{fin}}+\eta^{l+1} \tilde{\Gamma}^{(k)}\left(S_{R}^{[l]}\right)_{l+1, \mathrm{div}} \\
& +O\left(\eta^{l+2}\right) \\
\Gamma= & \Gamma\left(S_{R}^{[l]}\right)_{\mathrm{fin}}+\eta^{l+1}\left[\Gamma\left(S_{R}^{[l]}\right)_{l+1, \mathrm{div}}+s_{l+1}\right] \\
& +O\left(\eta^{l+2}\right) \\
\Gamma\left(S_{R}^{[l]}\right)_{l+1, \mathrm{div}}= & \tilde{\Gamma}^{(1)}\left(S_{R}^{[l]}\right)_{l+1, \mathrm{div}} \\
& +\chi \tilde{\Gamma}^{(2)}\left(S_{R}^{[l]}\right)_{l+1, \mathrm{div}}
\end{aligned}
$$

so that the functionals $\tilde{\Gamma}^{(k)}\left(S_{R}^{[l]}\right)_{l+1 \text {, div }}$ are local ones of arguments with the quantum numbers of the action $S_{\text {ext }}$ and contain divergent terms only (the minimal subtraction scheme). Then, as a consequence of the Eqs. (4.28), (4.29) (4.32), they satisfy the following equations,

$$
\begin{aligned}
& \left(\tilde{\Gamma}_{0}, \tilde{\Gamma}\left(S_{R}^{[l]}\right)_{l+1, \mathrm{div}}^{(1)}\right) \\
& -\int d x\left(\theta \frac{\delta}{\delta \mathcal{B}}\right) \tilde{\Gamma}\left(S_{R}^{[l]}\right)_{l+1, \operatorname{div}}^{(1)}=0, \\
& 2 \xi \frac{\partial}{\partial \xi} \tilde{\Gamma}\left(S_{R}^{[l]}\right)_{l+1, \operatorname{div}}^{(1)}=\left(\tilde{\Gamma}_{0}^{(1)}, \tilde{\Gamma}\left(S_{R}^{[l]}\right)_{l+1, \operatorname{div}}^{(2)}\right) \\
& -\left(\tilde{\Gamma}_{0}^{(2)}, \tilde{\Gamma}\left(S_{R}^{[l]}\right)_{l+1, \mathrm{div}}^{(1)}\right) \\
& -\int d x\left(\theta \frac{\delta}{\delta \mathcal{B}}\right) \tilde{\Gamma}\left(S_{R}^{[l]}\right)_{l+1, \mathrm{div}}^{(2)} \\
& +\int d x\left(\Omega_{m \mid}^{*} \frac{\delta}{\delta \Omega_{m \mid}^{*}}-\Omega_{m \mid} \frac{\delta}{\delta \Omega_{m \mid}}\right) \tilde{\Gamma}\left(S_{R}^{[l]}\right)_{l+1, \mathrm{div}}^{(1)}, \\
& \tilde{\Gamma}\left(S_{R}^{[l]}\right)_{l+1, \operatorname{div}}^{(k)} \overleftarrow{h^{\alpha}} \omega_{\alpha}=0 \\
& \tilde{\Gamma}\left(S_{R}^{[l]}\right)_{l+1, \operatorname{div}}^{(k)} \overleftarrow{T^{\alpha}}=0, \quad k=1,2
\end{aligned}
$$

Notice that the form of the Eqs. (5.31)-(5.34) does not depend on the label $l$.

By taking into account the quantum numbers, axial-, Poincare-, $T$-symmetries, the general expression for local functional $\tilde{\Gamma}\left(S_{R}^{[l]}\right)_{l+1, \text { div }}^{(2)}$, reads

$$
\begin{aligned}
\tilde{\Gamma}\left(S_{R}^{[l]}\right)_{l+1, \operatorname{div}}^{(2)}= & \int d x\left(q_{1, l+1} \mathcal{A}^{*} \mathcal{A}+q_{2, l+1} C^{*} C\right. \\
& \left.+q_{3, l+1} \psi^{*} \psi+q_{4, l+1} \bar{\psi}^{*} \bar{\psi}+q_{1, l+1}^{\prime} \mathcal{A}^{*} \mathcal{B}\right),
\end{aligned}
$$

where $q_{i, l+1}, i=1,2,3,4$, " $Z$ ", " $p$ " $q_{1, l+1}^{\prime}$ are arbitrary constants. Then, by using the Eq. (5.33) for $\tilde{\Gamma}\left(S_{R}^{[l]}\right)_{l+1 \text {,div }}^{(2)}$, we find that $q_{1, l+1}^{\prime}=0$. The final expression for $\tilde{\Gamma}\left(S_{R}^{[l]}\right)_{l+1, \operatorname{div}}^{(2)}$ has the form

$$
\begin{aligned}
\tilde{\Gamma}\left(S_{R}^{[l]}\right)_{l+1, \mathrm{div}}^{(2)}= & \int d x\left(q_{1, l+1} \mathcal{A}^{*} \mathcal{A}+q_{2, l+1} C^{*} C\right. \\
& \left.+q_{3, l+1} \psi^{*} \psi+q_{4, l+1} \bar{\psi}^{*} \bar{\psi}\right),
\end{aligned}
$$

Notice that the functional $\tilde{\Gamma}\left(S_{R}^{[l]}\right)_{l+1 \text {,div }}^{(2)}$ does not depend on the fields $\theta$ and $\mathcal{B}$.

With the expression (5.36) for $\tilde{\Gamma}\left(S_{R}^{[l]}\right)_{l+1 \text {,div }}^{(2)}$, the Eq. (5.32) reduces to the following one,

$$
\begin{aligned}
& 2 \xi \frac{\partial}{\partial \xi} \tilde{\Gamma}\left(S_{R}^{[l]}\right)_{l+1, \mathrm{div}}^{(1)}-\int d x\left[q_{1, l+1}\left(\mathcal{A} \frac{\delta}{\delta \mathcal{A}}-\mathcal{A}^{*} \frac{\delta}{\delta \mathcal{A}^{*}}\right)\right. \\
& \quad+q_{2, l+1}\left(C \frac{\delta}{\delta C}-C^{*} \frac{\delta}{\delta C^{*}}\right)+q_{3, l+1}\left(\psi \frac{\delta}{\delta \psi}-\psi^{*} \frac{\delta}{\delta \psi^{*}}\right) \\
& \left.\quad+q_{4, l+1}\left(\bar{\psi} \frac{\delta}{\delta \bar{\psi}}-\bar{\psi}^{*} \frac{\delta}{\delta \bar{\psi}^{*}}\right)\right] \tilde{\Gamma}_{0}^{(1)}=0 .
\end{aligned}
$$

\subsubsection{Solution to equation (5.31) for $\tilde{\Gamma}\left(S_{R}^{[l]}\right)_{l+1 \text {, div }}^{(1)}$}

Consider a solution to the Eq. (5.31) for the functional $\tilde{\Gamma}_{l+1, \mathrm{div}}^{(1)}=\tilde{\Gamma}\left(S_{R}^{[l]}\right)_{l+1, \mathrm{div}}^{(1)}$, represented in the form,

$$
\begin{aligned}
\tilde{\Gamma}_{l+1, \operatorname{div}}^{(1)}= & M_{\theta, l+1}+M_{\Omega^{*}, l+1}+M_{\psi, l+1}+M_{\mathcal{A B}, l+1}, \\
M_{\Omega^{*}, l+1}= & M_{\mathcal{A}^{*}, l+1}+M_{C^{*}, l+1}+M_{\psi^{*}, l+1} \\
& +M_{\bar{\psi}^{*}, l+1} .
\end{aligned}
$$

With this aim, we find first the general form of the functional $\tilde{\Gamma}_{l+1 \text {,div }}^{(1)}$, using the locality, the quantum numbers, axial-, Poincare-, $T$-symmetries and partially the gauge symmetry in the external field $\mathcal{B}$. In fact, all required calculations do copy ones performed in Sect. 3 when constructing the general form of the functional $\tilde{P}^{(1)}$ [see formulas (3.21)-(3.35) with the obvious replacements like $\left.\tilde{P}_{\theta}^{(1)} \rightarrow M_{\theta}\right]$. Here, we reproduce the final results only. The functional $M_{\theta, l+1}$ has the form 
$M_{\theta, l+1}=q_{5, l+1} \int d x \mathcal{A}_{\mu}^{* \alpha}(x) \theta_{\mu}^{\alpha}(x)=q_{5, l+1} \int d x \mathcal{A}^{*} \theta$.

For the functionals linear in antifields we find

$$
\begin{aligned}
M_{\mathcal{A}^{*}, l+1}= & \int d x\left[q_{6, l+1} \mathcal{A}_{\mu}^{* \alpha} D_{\mu}^{\alpha \beta}(\mathcal{B}) C^{\beta}\right. \\
& \left.+g q_{7, l+1 \beta \gamma}^{\alpha} \mathcal{A}_{\mu}^{* \alpha} \mathcal{A}_{\mu}^{\beta} C^{\gamma}\right], \\
M_{C^{*}, l+1}= & \int d x \frac{g}{2} q_{8, l+1 \beta \gamma}^{\alpha} C^{* \alpha} C^{\beta} C^{\gamma}, \\
M_{\psi^{*}, l+1}= & -\int d x g q_{9, l+1 j k}^{\alpha} \psi_{j}^{*} \psi_{k} C^{\alpha}, \\
M_{\bar{\psi}^{*}, l+1}= & \int d x g q_{10, l+1 k j}^{\alpha} \bar{\psi}_{j}^{*} \bar{\psi}_{k} C^{\alpha},
\end{aligned}
$$

where the constants " $q$ " satisfy the equations (we omit the lower case $l+1$ )

$$
\begin{aligned}
F_{\gamma \delta}^{\alpha} q_{7 \delta \sigma}^{\beta}-q_{7 \gamma \delta}^{\beta} F_{\delta \sigma}^{\alpha} & =f^{\alpha \beta \lambda} q_{7 \gamma \sigma}^{\lambda}, \\
F_{\gamma \delta}^{\alpha} q_{8 \delta \sigma}^{\beta}-q_{8 \gamma \delta}^{\beta} F_{\delta \sigma}^{\alpha} & =f^{\alpha \beta \delta} q_{8 \gamma \sigma}^{\delta}, \\
t_{j l}^{\alpha} q_{9 l k}^{\beta}-q_{9 j l}^{\beta} t_{l k}^{\alpha} & =f^{\alpha \beta \gamma} q_{9 j k}^{\gamma}, \\
t_{k l}^{\alpha} q_{10 l j}^{\beta}-q_{10 k l}^{\beta} \alpha_{l j}^{\alpha} & =f^{\alpha \beta \gamma} q_{10 k j}^{\gamma} .
\end{aligned}
$$

For the functionals $M_{\psi, l+1}$ we obtain

$$
\begin{aligned}
& M_{\psi, l+1}=\int d x\left[i q_{11, l+1} \bar{\psi} \gamma^{\mu} D_{\psi \mu}(\mathcal{B}) \psi\right. \\
& \left.\quad+i g q_{12, l+1, j k}^{\alpha} \bar{\psi}_{j} \gamma^{\mu} \mathcal{A}_{\mu}^{\alpha} \psi_{k}-m q_{13, l+1} \bar{\psi} \psi\right], \\
& t_{j r}^{\alpha} q_{12, l+1, r k}^{\beta}-q_{12, l+1, r l}^{\beta} t_{r k}^{\alpha}=f^{\alpha \beta \gamma} q_{12, l+1, j k}^{\gamma} .
\end{aligned}
$$

As the coefficient of the $\theta_{\mu}^{\alpha}$ term should be zero, it follows that the equation

$$
\begin{aligned}
& q_{5, l+1} \partial_{\mathcal{A}}\left(\Gamma_{0 \mathcal{A}^{*}}+\Gamma_{0 \psi}+\Gamma_{0 \mathcal{A B}}\right)+\partial_{\mathcal{A}}\left(M_{\mathcal{A}^{*}, l+1}+M_{\psi, l+1}\right. \\
& \left.\quad+M_{\mathcal{A B}, l+1}\right) \\
& \quad-\partial_{\mathcal{B}}\left(M_{\mathcal{A}^{*}, l+1}+M_{\psi, l+1}+M_{\mathcal{A B}, l+1}\right)=0
\end{aligned}
$$

holds. As the coefficient of the $\bar{\psi} \psi$ vertex of the Eq. (5.49) should be zero, it follows that

$q_{12, l+1, j k}^{\alpha}=q_{12, l+1} t_{j k}^{\alpha}, \quad q_{12, l+1}=q_{11, l+1}-q_{5, l+1}$.

In turn, as the coefficient of the $\mathcal{A}^{*} C$ vertex of the Eq. (5.49) should be zero, it follows that

$q_{7, l+1, \beta \gamma}^{\alpha}=q_{7, l+1} f^{\alpha \beta \gamma}, q_{7, l+1}=q_{6, l+1}-q_{5, l+1}$.

When inserting the expressions found for the $q_{12, l+1, j k}^{\alpha}$ and $q_{7, l+1, \beta \gamma}^{\alpha}$ coefficients into the Eq. (5.49), it reduces to the following one

$q_{5, l+1} \partial_{\mathcal{A}_{\mu}^{\alpha}} \Gamma_{0 \mathcal{A B}}+\partial_{\mathcal{A}_{\mu}^{\alpha}} M_{\mathcal{A B}, l+1}-\partial_{\mathcal{B}_{\mu}^{\alpha}} M_{\mathcal{A B}, l+1}=0$.
The general solution to the Eq. (5.52) reads

$M_{\mathcal{A B}, l+1}=-q_{5, l+1} \mathcal{A} \partial_{\mathcal{A}} \Gamma_{0 \mathcal{A B}}(V)+M_{1, l+1}(V), \quad V=\mathcal{A}+\mathcal{B}$,

where $M_{1, l+1}(V)$ stands for an arbitrary functional of $V$, at the moment.

In addition, insertion of the expression (5.51) for the $q_{7, l+1, \beta \gamma}^{\alpha}$ coefficient into the relation (5.40) yields

$M_{\mathcal{A}^{*}, l+1}=q_{6, l+1} \Gamma_{0 \mathcal{A}^{*}}(V)-q_{5, l+1} \mathcal{A} \partial_{\mathcal{A}} \Gamma_{0 \mathcal{A}^{*}}(V)$,

and insertion (5.50) for the $q_{12, l+1, j k}^{\alpha}$ coefficient into (5.47) gives the expression for $M_{\psi, l+1}$,

$M_{\psi, l+1}=q_{11, l+1} \Gamma_{0 \psi \mid 1}-q_{5, l+1} \mathcal{A} \partial_{\mathcal{A}} \Gamma_{0 \psi \mid 1}+q_{13, l+1} \Gamma_{0 \psi \mid 2}$.

At $\theta=0$ the Eq. (5.31) is reduced to the one

$$
\begin{gathered}
\int d x\left(\Gamma_{0 \Omega^{*}}+\Gamma_{0 \psi}+\Gamma_{0 \mathcal{A B}}\right)\left(\overleftarrow{\partial}_{\Omega} \partial_{\Omega^{*}}-\overleftarrow{\partial}_{\Omega^{*}} \partial_{\Omega}\right) \\
\left(M_{\Omega^{*}, l+1}+M_{\psi, l+1}+M_{\mathcal{A B}, l+1}\right)=0
\end{gathered}
$$

which is not more than linear in antifields.

As the coefficient of the $\mathcal{A}^{*} D(\mathcal{B}) C C$ vertex of the Eq. (5.56) should be zero, it follows that

$q_{8, l+1, \beta \gamma}^{\alpha}=q_{8, l+1} f^{\alpha \beta \gamma}, \quad q_{8, l+1}=q_{7, l+1}=q_{6, l+1}-q_{5, l+1}$.

Next, we consider the equations which follow from (5.56) for zero-valued antifields. They split into the two sets of equations. In the first set of equations,

$\Gamma_{0 \psi} \overleftarrow{\partial}_{\Omega} \partial_{\Omega^{*}} M_{\Omega^{*}, l+1}-\Gamma_{0 \Omega^{*}} \overleftarrow{\partial}_{\Omega^{*}} \partial_{\Omega} M_{\psi, l+1}=0$

all vertexes contain the spinor fields. In the second ones,

$\Gamma_{0 \mathcal{A B}} \overleftarrow{\partial} \mathcal{A}_{\mathcal{A}^{*}} M_{\mathcal{A}^{*}, l+1}-\Gamma_{0 \mathcal{A}^{*}} \overleftarrow{\partial}_{\mathcal{A}^{*}} \partial_{\mathcal{A}} M_{\mathcal{A B}, l+1}=0$

vertexes are constructed of the fields $\mathcal{A}, \mathcal{B}$ and their coordinate-derivatives only.

As the coefficient of the $\bar{\psi} \psi \partial_{\mu} C$ vertex of the Eq. (5.58) should be zero, it follows

$q_{9, l+1, j k}^{\alpha}=q_{9, l+1} t_{j k}^{\alpha}, \quad q_{9, l+1}=q_{6, l+1}-q_{5, l+1}$.

As the coefficient of the $\bar{\psi} \psi C$ vertex of the Eq. (5.58) should be zero, it follows the relation,

$q_{10, l+1, j k}^{\alpha}=q_{9, l+1, j k}^{\alpha}=q_{9, l+1} t_{j k}^{\alpha}$. 
When inserting the expressions (5.53) and (5.54) for $M_{\mathcal{A B}, l+1}$ and $M_{\mathcal{A}^{*}, l+1}$, respectively, into the Eq. (5.59), it reduces to the following equation,

$D(V) \partial_{V} M_{1, j+1}(V)=0 \Rightarrow M_{1, j+1}(V)=q_{14, l+1} \Gamma_{0 \mathcal{A B}}$.

Thus, the functional $\Gamma\left(S_{R}^{[l]}\right)_{l+1 \text {, div }}$ describing the " $Z$ ", " $p$ " $(l+1)$-loop divergences of the functional $\Gamma\left(S_{R}^{[l]}\right)$, has the form

$\Gamma\left(S_{R}^{[l]}\right)_{l+1, \operatorname{div}}=\tilde{\Gamma}^{(1)}\left(S_{R}^{[l]}\right)_{l+1, \operatorname{div}}+\chi \tilde{\Gamma}^{(2)}\left(S_{R}^{[l]}\right)_{l+1, \operatorname{div}}$,

where the functional $\tilde{\Gamma}^{(2)}\left(S_{R}^{[l]}\right)_{l+1 \text {, div }}$ is given by the expression (5.36). As for the functional $\tilde{\Gamma}^{(1)}\left(S_{R}^{[l]}\right)_{l+1 \text {, div }}$ we use the representation

$\tilde{\Gamma}\left(S_{R}^{[l]}\right)_{l+1, \mathrm{div}}^{(1)}=M_{\theta, l+1}+M_{\Omega^{*}, l+1}+M_{\psi, l+1}+M_{\mathcal{A B}, l+1}$,

where the functionals " $M$ ",

$$
\begin{aligned}
M_{\theta, l+1}= & q_{5, l+1} \Gamma_{0 \theta}, \\
M_{\mathcal{A}^{*}, l+1}= & q_{6, l+1} \Gamma_{0 \mathcal{A}^{*}}(V)-q_{5, l+1} \mathcal{A}_{\mathcal{A}} \Gamma_{0 \mathcal{A}^{*}}(V), \\
M_{C^{*}, l+1}= & \left(q_{6, l+1}-q_{5, l+1}\right) \Gamma_{0 C^{*}}, \\
M_{\psi^{*}, l+1}= & \left(q_{6, l+1}-q_{5, l+1}\right) \Gamma_{0 \psi^{*}}, \\
M_{\bar{\psi}^{*}, l+1}= & \left(q_{6, l+1}-q_{5, l+1}\right) \Gamma_{0 \bar{\psi}^{*}} \\
M_{\psi, l+1}= & q_{11, l+1} \Gamma_{0 \psi \mid 1}-q_{5, l+1} \mathcal{A} \partial_{\mathcal{A}} \Gamma_{0 \psi \mid 1} \\
& +q_{13, l+1} \Gamma_{0 \psi \mid 2} \\
M_{\mathcal{A B}, l+1}= & q_{14, l+1} \Gamma_{0 \mathcal{A B}}-q_{5, l+1} \mathcal{A}_{\mathcal{A}} \Gamma_{0 \mathcal{A B}},
\end{aligned}
$$

are represented in terms of the tree loop functionals " $\Gamma_{0}$ " (5.5)-(5.10).

\subsubsection{Solution to equation (5.37) for $\tilde{\Gamma}\left(S_{R}^{[l]}\right)_{l+1 \text {, div }}^{(1)}$}

When inserting the representation for the functional $\tilde{\Gamma}^{(1)}\left(S_{R}^{[l]}\right)_{l+1 \text {,div }}$ given by (5.64)-(5.71) into the Eq. (5.37), it takes the form of zero value for some linear combinations of structures appeared in the right-hand side of formulas (5.65)(5.71).

As the coefficient of the $\theta_{\mu}^{\alpha}$ term should be zero, it follows that

$q_{1, l+1}=-2 \xi \dot{q}_{5, l+1}$.

Then, as the coefficient of any antifield term should be zero, it follows

$q_{2, l+1}=2 \xi\left(\dot{q}_{6, l+1}-\dot{q}_{5, l+1}\right)$.

When the relations (5.72) and (5.73) hold, then the Eq. (5.37) reduces to the two equations having obvious solutions

$$
\begin{aligned}
& 2 \xi \dot{q}_{14, l+1} \Gamma_{0 \mathcal{A B}}=0 \Rightarrow \dot{q}_{14, l+1}=0, \\
& 2 \xi \dot{q}_{11, l+1} \Gamma_{0 \psi \mid 1}+2 \xi \dot{q}_{11, l+1} \Gamma_{0 \psi \mid 2} \\
& \quad-\left(q_{3, l+1}+q_{4, l+1}\right)\left(\Gamma_{0 \psi \mid 1}+\Gamma_{0 \psi \mid 2}\right)=0 \Rightarrow \\
& 2 \xi \dot{q}_{11, l+1}=2 \xi \dot{q}_{13, l+1}=q_{3, l+1}+q_{4, l+1} .
\end{aligned}
$$

It is convenient to introduce new parameters $q_{15, l+1}$ and $q_{16, l+1}$,

$q_{15, l+1}=q_{13, l+1}-q_{11, l+1}, \quad 2 q_{16, l+1}=q_{3, l+1}-q_{4, l+1}$,

in terms of which the Eq. (5.76) rewrites as

$\dot{q}_{15, l+1}=0, \quad q_{3, l+1}=\xi \dot{q}_{11, l+1}+q_{16, l+1}$,

$q_{4, l+1}=\xi \dot{q}_{11, l+1}-q_{16, l+1}$.

\subsection{Finiteness of $\Gamma$ to $(1+1)$-loop approximation}

Now, let us prove that one can chose the renormalization constants in such a way as to make the effective action finite to the $(l+1)$-loop approximation. To this end, we consider the divergent part of the effective action $\Gamma, \Gamma_{l+1 \text {,div }}$, described by the Eq. (5.29),

$$
\Gamma_{l+1, \mathrm{div}}=\Gamma\left(S_{R}^{[l]}\right)_{l+1, \mathrm{div}}+s_{l+1}=\Gamma_{l+1, \mathrm{div}}^{(1)}+\chi \Gamma_{l+1, \mathrm{div}}^{(2)} .
$$

For the functionals $\Gamma_{l+1 \text {,div }}^{(1)}, \Gamma_{l+1 \text {,div }}^{(2)}$ we have the representations

$$
\begin{aligned}
\Gamma_{l+1, \mathrm{div}}^{(1)}= & \Gamma\left(S_{R}^{[l]}\right)_{l+1, \mathrm{div}}^{(1)}+s_{l+1}^{(1)} \\
= & \Gamma_{\theta, l+1, \mathrm{div}}+\Gamma_{\Omega^{*}, l+1, \mathrm{div}} \\
& +\Gamma_{\psi, l+1, \mathrm{div}}+\Gamma_{\mathcal{A} \mathcal{B}, l+1, \mathrm{div}}, \\
\Gamma_{\theta, l+1, \mathrm{div}}= & \left(q_{5, l+1}+z_{5, l+1}\right) \Gamma_{0 \theta}, \\
\Gamma_{\mathcal{A}^{*} \theta, l+1, \mathrm{div}}= & \left(q_{6, l+1}+z_{6, l+1}\right) \Gamma_{0 \mathcal{A}^{*}}(V) \\
& -\left(q_{5, l+1}+z_{5, l+1}\right) \mathcal{A} \partial_{\mathcal{A}} \Gamma_{0 \mathcal{A}^{*}}(V), \\
\Gamma_{C^{*}, l+1, \mathrm{div}}= & \left(q_{6, l+1}+z_{6, l+1}-q_{5, l+1}-z_{5, l+1}\right) \Gamma_{0 C^{*},} \\
\Gamma_{\psi^{*}, l+1, \mathrm{div}}= & \left.q_{6, l+1}+z_{6, l+1}-q_{5, l+1}-z_{5, l+1}\right) \Gamma_{0 \psi^{*}}, \\
\Gamma_{\bar{\psi}^{*}, l+1, \mathrm{div}}= & \left.q_{6, l+1}+z_{6, l+1}-q_{5, l+1}-z_{5, l+1}\right) \Gamma_{0 \bar{\psi}^{*}}, \\
\Gamma_{\psi, l+1, \mathrm{div}}= & \left(q_{11, l+1}+z_{11, l+1}\right) \Gamma_{0 \psi \mid 1} \\
& -\left(q_{5, l+1}+z_{5, l+1}\right) \mathcal{A} \partial_{\mathcal{A}} \Gamma_{0 \psi \mid 1}+ \\
& +\left(q_{11, l+1}+z_{11, l+1}\right. \\
& \left.+q_{15, l+1}+z_{15, l+1}\right) \Gamma_{0 \psi \mid 2}, \\
\Gamma_{\mathcal{A} \mathcal{B}, l+1, \mathrm{div}}= & \left(q_{14, l+1}+z_{14, l+1}\right) \Gamma_{0 \mathcal{A}} \mathcal{B} \\
& -\left(q_{5, l+1}+z_{5, l+1}\right) \mathcal{A} \partial_{\mathcal{A}} \Gamma_{0 \mathcal{A}}, \\
\Gamma_{l+1, \mathrm{div}}^{(2)}= & \Gamma\left(S_{R}^{[l]}\right)_{l+1, \mathrm{div}}^{(2)}+s_{l+1}^{(2)} \\
= & \int d x\left[2 \xi\left(\dot{q}_{5, l+1}+\dot{z}_{5, l+1}\right) \mathcal{A}^{*} \mathcal{A}+\right.
\end{aligned}
$$




$$
\begin{aligned}
& +2 \xi\left(\dot{q}_{6, l+1}+\dot{z}_{6, l+1}-\dot{q}_{5, l+1}-\dot{z}_{5, l+1}\right) C^{*} C \\
& +\left(\xi \dot{q}_{11, l+1}+\xi \dot{z}_{11, l+1}+q_{16, l+1}+\right. \\
& \left.+z_{16, l+1}\right) \psi^{*} \psi+\left(\xi \dot{q}_{11, l+1}+\xi \dot{z}_{11, l+1}\right. \\
& \left.\left.-q_{16, l+1}-z_{16, l+1}\right) \bar{\psi}^{*} \bar{\psi}\right] .
\end{aligned}
$$

It follows from the formulas (5.79)-(5.88), that the choice of the parameters $z_{i, l+1}$ in the form " $Z$ ", " $p$ "

$$
\begin{aligned}
z_{i, l+1} & =z_{i, l+1, \mathrm{fin}}, \quad z_{i, l+1, \mathrm{fin}}=-q_{i, l+1}, \\
i & =5,6,11,14,15,16,
\end{aligned}
$$

provides for zero-valued coefficients to the $(l+1)$-loop divergences,

$$
\begin{aligned}
\left.\Gamma_{l+1, \operatorname{div}}\right|_{z_{i, l+1}=z_{i, l+1, \mathrm{fin}}} & =0 \\
\left.\Gamma_{l+1}\right|_{z_{i, l+1}=z_{i, l+1, \mathrm{fin}}} & =\Gamma_{l+1, \mathrm{fin} .}
\end{aligned}
$$

Notice that the choice of parameters $z_{i, l+1, \text { fin }}$ is unique within the minimal subtraction scheme.

\section{$5.4(1+2)-$ loop approximation}

The renormalization of $S_{R}$ to the $(l+1)$-loop approximation allows one to construct the effective action $\Gamma$, finite to that approximation; however it does not satisfy exactly the extended master-equation and the gauge dependence equation, by itself. We show the possibility to complete the renormalization constants of the action $S_{R}$ with the help of the $(l+2)$-loop approximation, so that it will satisfy the equations mentioned to the $(l+1)$-loop approximation and, in its turn, the corresponding effective action, finite to the $(l+1)$ loop approximation, will satisfy the set of Eqs. (2.21)-(2.25) to that approximation.

Indeed, we represent the action $S_{R}$ as

$S_{R}=S_{R}^{[l+1]}+\eta^{l+2} s_{l+2}+O\left(\eta^{l+3}\right)$,

where $S_{R}^{[l+1]}$ is the action $S_{R}$ with independent parameters " $Z$ ", " $p$ " $Z_{i}$ replaced by $Z_{i}^{[l+1]}$, and $s_{l+2}$ is equal to

$s_{l+2}=s_{l+2}^{(1)}+\chi s_{l+2}^{(2)}$,

where

$$
\begin{aligned}
s_{l+2}^{(1)}= & s_{\theta, l+2}+s_{\Omega^{*}, l+2}+s_{\psi, l+2}+s_{\mathcal{A B}, l+2}, \\
s_{\theta, l+2}= & z_{5, l+2} \Gamma_{0 \theta}, \\
s_{\mathcal{A}^{*}, l+2}= & z_{6, l+2} \Gamma_{0 \mathcal{A}^{*}}-z_{5, l+2} \mathcal{A} \partial_{\mathcal{A}} \Gamma_{0 \mathcal{A}^{*}}, \\
s_{C^{*}, l+2}= & \left(z_{6, l+1}-z_{5, l+2}\right) \Gamma_{0 C^{*}}, \\
s_{\psi^{*}, l+2}= & \left(z_{6, l+2}-z_{5, l+2}\right) \Gamma_{0 \psi^{*}}, \\
s_{\bar{\psi}^{*}, l+2}= & \left(z_{6, l+2}-z_{5, l+2}\right) \Gamma_{0 \bar{\psi}^{*}}{ }^{*}, \\
s_{\psi, l+2}= & z_{11, l+2} \Gamma_{0 \psi \mid 1}-z_{5, l+2} \mathcal{A}_{\mathcal{A}} \Gamma_{0 \psi \mid 1} \\
& +\left(z_{11, l+2}+z_{15, l+2}\right) \Gamma_{0 \psi \mid 2}, \\
s_{\mathcal{A B}, l+2}= & z_{14, l+2} \Gamma_{0 \mathcal{A B}}-z_{5, l+2} \mathcal{A} \partial_{\mathcal{A}} \Gamma_{0 \mathcal{A B}},
\end{aligned}
$$

$$
\begin{aligned}
s_{l+2}^{(2)}= & \int d x\left[2 \xi \dot{z}_{5, l+2} \mathcal{A}^{*} \mathcal{A}\right. \\
& +2 \xi\left(\dot{z}_{6, l+2}-\dot{z}_{5, l+2}\right) C^{*} C+ \\
& +\xi\left(\dot{z}_{11, l+2}+z_{16, l+2}\right) \psi^{*} \psi+ \\
& \left.+\xi\left(\dot{z}_{11, l+2}-z_{16, l+2}\right) \bar{\psi}^{*} \bar{\psi}\right], \\
& \dot{z}_{14, l+2}=\dot{z}_{15, l+2}=0 .
\end{aligned}
$$

Notice that the action $S_{R}^{[l+1]}$ satisfies the Eqs. (2.21)(2.25).

Further calculations and consequences from them do copy exactly the results of the previous subsection with the natural replacement $l+1 \rightarrow l+2$.

Also, it is obvious that the procedure of divergence compensations discussed can be applied to the case $l=0$ so that by using the loop induction method in Feynman diagrams for the functional $\Gamma$, we arrive at the following statement: for the $l$-loop approximation $\Gamma^{[l]}$, where $l$ is arbitrary positive integer,

$\Gamma^{[l]}=\sum_{n=0}^{l} \eta^{n} \Gamma_{n}$,

of the functional $\Gamma$ defined by the relations (4.1), (4.4), there exists the uniquely defined parameters $Z_{i}^{[l]}, i=$ $5,6,11,14,15,16$,

$\dot{Z}_{14}^{[l]}=0, \quad \dot{Z}_{15}^{[l]}=0, \quad \forall l \geq 0$,

such that the functional $\Gamma^{[l]}$ does not contain divergences and $\Gamma$ satisfies the Eqs. (4.6)-(4.9).

\section{Relations between parameters of $S_{R}$ and standard renormalization constants}

In that section we find relations between some parameters of the action $S_{R}$ and the standard renormalization constants. Within the expression for $S_{R}$, we restrict ourselves only by desired vertexes in symbolic notation

$$
\begin{aligned}
S_{R}= & \int d x\left(Z_{14} Z_{5}^{-2} \partial A \partial A+g Z_{14} Z_{5}^{-3} A^{2} \partial A+Z_{11} \bar{\psi} \partial \psi\right. \\
& \left.+m Z_{13} \bar{\psi} \psi+\ldots\right),
\end{aligned}
$$

where the ellipsis means the rest vertexes. As the propagators of fields $A$ and $\psi$ are finite, they should be considered as renormalized fields. Then, we find:

$Z_{A}=Z_{14}^{1 / 2} Z_{5}^{-1}, \quad Z_{\psi}=Z_{11}^{1 / 2}$,

where $Z_{A}$ and $Z_{\psi}$ are the renormalization constants of the bare fields $A_{0}$ and $\psi_{0}$. The coefficient of the second vertex in the expression (6.1) gives the renormalization for vertex $A^{3}$,

$Z_{A^{3}}=Z_{14} Z_{5}^{-3} \Rightarrow g_{0}=Z_{g} g, Z_{g}=Z_{A^{3}} Z_{A}^{-3}=Z_{14}^{-1 / 2}$. 
The coefficient of the forth vertex in the expression (6.1) gives the renormalization for vertex $\bar{\psi} \psi$,

$$
\begin{aligned}
Z_{\bar{\psi} \psi} & =Z_{13} \Rightarrow m_{0}=Z_{m} m, \\
Z_{m} & =Z_{\bar{\psi} \psi} Z_{\psi}^{-2}=Z_{13} / Z_{11}=Z_{15} .
\end{aligned}
$$

It follows from the Eq. (5.103) that the renormalization constants of physical parameters $g$ and $m$ do not depend on gauge,

$\partial_{\xi} Z_{g}=0, \quad \partial_{\xi} Z_{m}=0$.

\section{Summary}

In the present paper, within the background field formalism, it is studied the renormalization procedure and the gauge dependence of the theory of Yang-Mills fields interacting with a multiplet of massive spinor fields. It is shown that the extension of the Faddeev-Popov action with extra fields and parameters allows one to establish the multiplicative character of the renormalizability. The proofs given above are based on the possibility to expand the effective action in loops, as well as to use the minimal subtraction scheme as to eliminate divergences. It is a new and important result that the renormalization constant of the mass parameter is shown to be gauge-independent.

Acknowledgements The work of I.A. Batalin and I.V. Tyutin is supported in part by the RFBR Grant 17-02-00317. The work of P.M. Lavrov is supported partially by the Ministry of Education and Science of the Russian Federation, Grant 3.1386.2017 and by the RFBR Grant 18-02-00153.

Open Access This article is distributed under the terms of the Creative Commons Attribution 4.0 International License (http://creativecomm ons.org/licenses/by/4.0/), which permits unrestricted use, distribution, and reproduction in any medium, provided you give appropriate credit to the original author(s) and the source, provide a link to the Creative Commons license, and indicate if changes were made.

Funded by SCOAP ${ }^{3}$.

\section{References}

1. C.N. Yang, R.L. Mills, Considerations of isotopic spin and isotopic gauge invariance. Phys. Rev. 96, 191 (1954)

2. L.D. Faddeev, V.N. Popov, Feynman diagrams for the Yang-Mills field. Phys. Lett. B 25, 29 (1967)
3. C. Becchi, A. Rouet, R. Stora, The abelian Higgs Kibble model, unitarity of the $S$-operator. Phys. Lett. B 52, 344 (1974)

4. I.V. Tyutin, Gauge invariance in field theory and statistical physics in operator formalism, Lebedev Inst. (1975) (preprint N 39)

5. J. Zinn-Justin, Renormalization of gauge theories, in Trends in elementary particle theory, lecture notes in physics, vol. 37, ed. by $\mathrm{H}$. Rollnik, K. Dietz (Springer, Berlin, 1975)

6. J.C. Taylor, Ward identities and charge renormalization of the Yang-Mills field. Nucl. Phys. B 33, 436 (1971)

7. A.A. Slavnov, Ward identities in gauge theories. Theor. Math. Phys. 10, 99-107 (1972)

8. I.A. Batalin, G.A. Vilkovisky, Gauge algebra and quantization. Phys. Lett. B 102, 27 (1981)

9. I.A. Batalin, G.A. Vilkovisky, Quantization of gauge theories with linearly dependent generators. Phys. Rev. D 28, 2567 (1983)

10. R. Jackiw, Functional evaluation of the effective potential. Phys. Rev. D 9, 1686 (1974)

11. L. Dolan, R. Jackiw, Gauge invariant signal for gauge symmetry breaking. Phys. Rev. D 9, 2904 (1974)

12. N.K. Nielsen, On the gauge dependence of spontaneous symmetry breaking in gauge theories. Nucl. Phys. B 101, 173 (1975)

13. R. Fukuda, T. Kugo, Gauge invariance in the effective action and potential. Phys. Rev. D 13, 3469 (1976)

14. P.M. Lavrov, I.V. Tyutin, On the structure of renormalization in gauge theories. Sov. J. Nucl. Phys. 34, 156 (1981)

15. P.M. Lavrov, I.V. Tyutin, On the generating functional for the vertex functions in Yang-Mills theories. Sov. J. Nucl. Phys. 34, 474 (1981)

16. N.K. Nielsen, Removing the gauge parameter dependence of the effective potential by a field redefinition. Phys. Rev. D 90, 036008 (2014)

17. A.D. Plancencia, C. Tamarit, Convexity, gauge dependence and tunneling rates. JHEP 1610, 099 (2016)

18. B.L. Voronov, P.M. Lavrov, I.V. Tyutin, Canonical transformations and the gauge dependence in general gauge theories. Sov. J. Nucl. Phys. 36, 292 (1982)

19. I.A. Batalin, P.M. Lavrov, I.V. Tyutin, Finite anticanonical transformations in field-antifield formalism. Eur. Phys. J. C 75, 270 (2015)

20. H. Kluberg-Stern, J.B. Zuber, Renormalization of non-Abelian gauge theories in a background-field gauge. I. Green's functions. Phys. Rev. D 12, 482 (1975)

21. B.S. DeWitt, Dynamical theory of groups and fields (Gordon and Breach, New York, 1965)

22. B.S. DeWitt, Quantum theory of gravity. II. The manifestly covariant theory. Phys. Rev. 162, 1195 (1967)

23. L.F. Abbott, The background field method beyond one loop. Nucl. Phys. B 185, 189 (1981) 